$\underline{\text { Preprint typeset in JHEP style - HYPER VERSION }}$

arXiv:0807.2589

UAB-FT-2008-652

CERN-TH/2008-155

SLAC-PUB-13307

\title{
New observables in the decay mode $\bar{B}_{d} \rightarrow \bar{K}^{* 0} \ell^{+} \ell^{-}$
}

\author{
Ulrik Egede \\ Imperial College London, London SW7 2AZ, United Kingdom \\ E-mail: U.Egede@imperial.ac.uk

\section{Tobias Hurth} \\ CERN, Dept. of Physics, Theory Division, CH-1211 Geneva 23, Switzerland* \\ E-mail: Tobias.Hurth@cern.ch

\section{Joaquim Matias} \\ IFAE, Universitat Autònoma de Barcelona, 08193 Bellaterra, Barcelona, Spain \\ E-mail: matias@ifae.es

\section{Marc Ramon} \\ IFAE, Universitat Autònoma de Barcelona, 08193 Bellaterra, Barcelona, Spain \\ E-mail: mramon@ifae.es
}

\section{Will Reece}

Imperial College London, London SW7 2AZ, United Kingdom

E-mail: w.reece06@imperial.ac.ul

ABSTRACT: We discuss the large set of observables available from the angular distributions of the decay $\bar{B}_{d} \rightarrow \bar{K}^{* 0} \ell^{+} \ell^{-}$. We present a NLO analysis of all observables based on the QCD factorization approach in the low-dilepton mass region and an estimate of $\Lambda / m_{b}$ corrections. Moreover, we discuss their sensitivity to new physics. We explore the experimental sensitivities at $\mathrm{LHC} b\left(10 \mathrm{fb}^{-1}\right)$ and SuperLHC $b\left(100 \mathrm{fb}^{-1}\right)$ based on a fullangular fit method and explore the sensitivity to right handed currents. We also show that the previously discussed transversity amplitude $A_{T}^{(1)}$ cannot be measured at the LHC $b$ experiment or at future $B$ factory experiments as it requires a measurement of the spin of the final state particles.

Keywords: B-Physics, Rare Decays.

\footnotetext{
*Also SLAC, Stanford University, Stanford, CA 94309, USA.
} 


\section{Contents}

1. Introduction

2. Differential decay distribution 2

3. $K^{*}$ spin amplitudes

4. Theoretically clean observables 6

4.1 General criteria 6

4.2 Observables 0

4.3 The problem with $\boldsymbol{A}_{\boldsymbol{T}}^{(\mathbf{1})}$ 8

5. Method to calculate experimental sensitivity 9

$5.1 \bar{B}_{d} \rightarrow \bar{K}^{* 0} \ell^{+} \ell^{-}$decay model 9

5.2 Full angular fit 10

5.3 Comparisons with fits to projections 11

6. Phenomenological analysis 13

6.1 Preliminaries 13

6.2 Results

7. Summary 20

A. Kinematics 23

B. Theoretical framework 24

G. NLO corrections to the spin amplitudes 25

\section{Introduction}

A major aim of particle physics in the LHC era is the discovery of new degrees of freedom at the $\mathrm{TeV}$ energy scale which might contribute to our understanding of the origin of electroweak symmetry breaking. Rare $B$ and kaon decays (for reviews see [1, 2, 23]) representing loop-induced processes are highly sensitive probes for new degrees of freedom beyond the Standard Model (SM) and will be used when making indirect searches for these unknown effects. It is well-known that the indirect constraints on new physics (NP) from the present flavour data indicate a NP scale much higher than the electroweak scale when such new effects are naturally parameterised by higher-dimensional operators. Thus, if there is NP at the electroweak scale, then its flavour structure has to be highly non-trivial and the 
experimental measurement of flavour-violating couplings is mandatory. This 'flavour problem', namely why flavour-changing neutral currents are suppressed, has to be solved by any NP scenario at the electroweak scale.

In this article we discuss theoretical and experimental preparations for an indirect NP search using the rare decay $\bar{B}_{d} \rightarrow \bar{K}^{* 0} \mu^{+} \mu^{-}$. This exclusive decay was first observed at Belle [4]. It offers a rich phenomenology of various kinematical distributions beyond the measurement of the branching ratio. Some experimental analyses of those angular distributions are already presented by the $B$ factories [5, 6, 0, 8, 9] but only the large increase in statistics at LHC $b$ [10, 11, 12] for $\bar{B}_{d} \rightarrow \bar{K}^{* 0} \mu^{+} \mu^{-}$will make much higher precision measurements possible. There are also great opportunities at the future (Super-) $B$ factories in this respect 13, 14, 15, 16. A careful choice of observables needs to be made to take full advantage of this exclusive decay as only in certain ratios such as $C P$ and forward-backward asymmetries, the hadronic uncertainties cancel out in specific observables making such ratios the only observables that are sensitive to NP. In this respect the by now standard theoretical tools like QCD factorization (QCDf) [17] and its quantum field theoretical formulation, soft-collinear effective theory (SCET), are crucial. They imply form factor relations which simplify the theoretical structure of various kinematical distributions such that at least at the leading order (LO) level any hadronic uncertainties cancel out. A well-known example of this is the zero-crossing of the forward-backward asymmetry.

We construct new observables of this kind in the $\bar{B}_{d} \rightarrow \bar{K}^{* 0} \mu^{+} \mu^{-}$decay which have very small theoretical uncertainties and good experimental resolution. Moreover, it is possible to design the new observables for a specific kind of NP operator within the model independent analysis using the effective field theory approach.

Previously proposed angular distributions and $C P$ violating observables in $\bar{B}_{d} \rightarrow$ $\bar{K}^{* 0} \mu^{+} \mu^{-}$are reviewed in Ref. [18, 15], and more recently QCDf analyses of such angular distributions [19, 20] and $C P$ violating observables [21] were presented.

The paper is organised as follows: in Sec. 2 we recall the differential decay distribution in the $\bar{B}_{d} \rightarrow \bar{K}^{* 0} \mu^{+} \mu^{-}$decay; in Sec. 3 we recall the basic theoretical formulae which are crucial for our construction of new observables; in Sec. 4 we discuss the basic properties and symmetries of potential observables and propose a new set of observables which are sensitive to new right-handed currents and we also discuss the previously proposed quantity $A_{T}^{(1)}$; in Sec. 5 we explain our method to calculate the experimental sensitivity obtainable with the statistics of $\mathrm{LHC} b$ to new and old observables; and finally in Sec. 6 we present our phenomenological analysis, in particular we analyse the theoretical and experimental sensitivity to NP. We also comment very briefly on recent BABAR measurements of certain angular distributions. In appendices we make angular definitions explicit, provide the theoretical framework for the derivation of the spin amplitudes, and present the theoretical NLO expressions.

\section{Differential decay distribution}

The decay $\bar{B}_{d} \rightarrow \bar{K}^{* 0} \ell^{+} \ell^{-}$with $\bar{K}^{* 0} \rightarrow K^{-} \pi^{+}$on the mass shell, is completely described 
by four independent kinematic variables, the lepton-pair invariant mass squared, $q^{2}$, and the three angles $\theta_{l}, \theta_{K}, \phi$. Summing over the spins of the final particles, the differential decay distribution of $\bar{B}_{d} \rightarrow \bar{K}^{* 0} \ell^{+} \ell^{-}$can be written as

$$
\frac{d^{4} \Gamma_{\bar{B}_{d}}}{d q^{2} d \theta_{l} d \theta_{K} d \phi}=\frac{9}{32 \pi} I\left(q^{2}, \theta_{l}, \theta_{K}, \phi\right) \sin \theta_{l} \sin \theta_{K},
$$

with the physical region of phase space $4 m_{l}^{2} \leqslant q^{2} \leqslant\left(m_{B}-m_{K^{*}}\right)^{2}$ and

$$
\begin{aligned}
I= & I_{1}+I_{2} \cos 2 \theta_{l}+I_{3} \sin ^{2} \theta_{l} \cos 2 \phi+I_{4} \sin 2 \theta_{l} \cos \phi+I_{5} \sin \theta_{l} \cos \phi+ \\
& +I_{6} \cos \theta_{l}+I_{7} \sin \theta_{l} \sin \phi+I_{8} \sin 2 \theta_{l} \sin \phi+I_{9} \sin ^{2} \theta_{l} \sin 2 \phi .
\end{aligned}
$$

The $I_{i}$ depend on products of the seven complex $K^{*}$ spin amplitudes, $A_{\perp L / R}, A_{\| L / R}$, $A_{0 L / R}, A_{t}$ (see next section) with each of these a function of $q^{2} . A_{t}$ is related to the time-like component of the virtual $K^{*}$, which does not contribute in the case of massless leptons and can be neglected if the lepton mass is small in comparison to the mass of the lepton pair. We will consider this case in our present analysis. For $m_{l}=0$, one finds [22, 23, 24, 25]:

$$
\begin{aligned}
& I_{1}=\frac{3}{4}\left(\left|A_{\perp L}\right|^{2}+\left|A_{\| L}\right|^{2}+(L \rightarrow R)\right) \sin ^{2} \theta_{K}+\left(\left|A_{0 L}\right|^{2}+\left|A_{0 R}\right|^{2}\right) \cos ^{2} \theta_{K} \\
& \equiv a \sin ^{2} \theta_{K}+b \cos ^{2} \theta_{K} \\
& I_{2}=\frac{1}{4}\left(\left|A_{\perp L}\right|^{2}+\left|A_{\| L}\right|^{2}\right) \sin ^{2} \theta_{K}-\left|A_{0 L}\right|^{2} \cos ^{2} \theta_{K}+(L \rightarrow R) \\
& \equiv c \sin ^{2} \theta_{K}+d \cos ^{2} \theta_{K}, \\
& I_{3}=\frac{1}{2}\left[\left(\left|A_{\perp L}\right|^{2}-\left|A_{\| L}\right|^{2}\right) \sin ^{2} \theta_{K}+(L \rightarrow R)\right] \equiv e \sin ^{2} \theta_{K}, \\
& I_{4}=\frac{1}{\sqrt{2}}\left[\operatorname{Re}\left(A_{0 L} A_{\| L}^{*}\right) \sin 2 \theta_{K}+(L \rightarrow R)\right] \equiv f \sin 2 \theta_{K}, \\
& I_{5}=\sqrt{2}\left[\operatorname{Re}\left(A_{0 L} A_{\perp L}^{*}\right) \sin 2 \theta_{K}-(L \rightarrow R)\right] \equiv g \sin 2 \theta_{K}, \\
& I_{6}=2\left[\operatorname{Re}\left(A_{\| L} A_{\perp L}^{*}\right) \sin ^{2} \theta_{K}-(L \rightarrow R)\right] \equiv h \sin ^{2} \theta_{K}, \\
& I_{7}=\sqrt{2}\left[\operatorname{Im}\left(A_{0 L} A_{\| L}^{*}\right) \sin 2 \theta_{K}-(L \rightarrow R)\right] \equiv j \sin 2 \theta_{K}, \\
& I_{8}=\frac{1}{\sqrt{2}}\left[\operatorname{Im}\left(A_{0 L} A_{\perp L}^{*}\right) \sin 2 \theta_{K}+(L \rightarrow R)\right] \equiv k \sin 2 \theta_{K}, \\
& I_{9}=\left[\operatorname{Im}\left(A_{\| L}^{*} A_{\perp L}\right) \sin ^{2} \theta_{K}+(L \rightarrow R)\right] \equiv m \sin ^{2} \theta_{K} .
\end{aligned}
$$

The exact equations presented here depend on the definition of the angles which we for this reason have made explicit in Appendix A.

From comparing the amplitude terms in Eq. (2.3), we see that $a=3 c$ and $b=-d$ thus leaving nine independent parameters which can be fixed experimentally in a full angular fit. Assuming massless leptons in the theory we have on the other hand 12 parameters from the six complex $\bar{K}^{* 0}$ spin amplitudes, $A_{\perp L / R}, A_{\| L / R}, A_{0 L / R}$. See Sec. $\mathbb{t}$ for an analysis of the apparent mismatch between the 9 and 12 parameters. 


\section{3. $K^{*}$ spin amplitudes}

The six complex $K^{*}$ spin amplitudes under the assumption of massless leptons are related to the well-known helicity amplitudes (used for example in [23, 24, 26]) through

$$
A_{\perp, \|}=\left(H_{+1} \mp H_{-1}\right) / \sqrt{2}, \quad A_{0}=H_{0} .
$$

The amplitudes describe the $B \rightarrow K \pi$ transition and can be parameterised in terms of the seven $B \rightarrow K^{*}$ form factors by means of a narrow-width approximation. They also depend on the short-distance Wilson coefficients $C_{i}$ corresponding to the various operators of the

effective electroweak Hamiltonian. The precise definitions of the form factors and of the effective operators are given in Appendix B. One obtains 19

$$
\begin{gathered}
A_{\perp L, R}=N \sqrt{2} \lambda^{1 / 2}\left[\left(\mathcal{C}_{9}^{(\mathrm{eff})} \mp \mathcal{C}_{10}\right) \frac{V\left(q^{2}\right)}{m_{B}+m_{K^{*}}}+\frac{2 m_{b}}{q^{2}}\left(\mathcal{C}_{7}^{(\mathrm{eff})}+\mathcal{C}_{7}^{\prime(\mathrm{eff})}\right) T_{1}\left(q^{2}\right)\right] \\
A_{\| L, R}=-N \sqrt{2}\left(m_{B}^{2}-m_{K^{*}}^{2}\right)\left[\left(\mathcal{C}_{9}^{(\mathrm{eff})} \mp \mathcal{C}_{10}\right) \frac{A_{1}\left(q^{2}\right)}{m_{B}-m_{K^{*}}}+\frac{2 m_{b}}{q^{2}}\left(\mathcal{C}_{7}^{(\mathrm{eff})}-\mathcal{C}_{7}^{\prime(\mathrm{eff})}\right) T_{2}\left(q^{2}\right)\right] \\
A_{0 L, R}=-\frac{N}{2 m_{K^{*}} \sqrt{q^{2}}} \times \\
\times\left[( \mathcal { C } _ { 9 } ^ { ( \mathrm { eff } ) } \mp \mathcal { C } _ { 1 0 } ) \left\{\left(m_{B}^{2}-m_{K^{*}}^{2}-q^{2}\right)\left(m_{B}+m_{K^{*}}\right) A_{1}\left(q^{2}\right)-\right.\right. \\
\left.\quad-\lambda \frac{A_{2}\left(q^{2}\right)}{m_{B}+m_{K^{*}}}\right\}+ \\
+2 m_{b}\left(\mathcal{C}_{7}^{(\mathrm{eff})}-\mathcal{C}_{7}^{\prime(\mathrm{eff})}\right)\left\{\left(m_{B}^{2}+3 m_{K^{*}}^{2}-q^{2}\right) T_{2}\left(q^{2}\right)-\right. \\
\left.\left.-\frac{\lambda}{m_{B}^{2}-m_{K^{*}}^{2}} T_{3}\left(q^{2}\right)\right\}\right]
\end{gathered}
$$

where

$$
\lambda=m_{B}^{4}+m_{K^{*}}^{4}+q^{4}-2\left(m_{B}^{2} m_{K^{*}}^{2}+m_{K^{*}}^{2} q^{2}+m_{B}^{2} q^{2}\right)
$$

and

$$
N=\sqrt{\frac{G_{F}^{2} \alpha^{2}}{3 \cdot 2^{10} \pi^{5} m_{B}^{3}}\left|V_{t b} V_{t s}^{*}\right|^{2} q^{2} \lambda^{1 / 2} \sqrt{1-\frac{4 m_{l}^{2}}{q^{2}}}} .
$$

The crucial theoretical input we use in our analysis is the observation that in the limit where the initial hadron is heavy and the final meson has a large energy 27] the hadronic form factors can be expanded in the small ratios $\Lambda_{\mathrm{QCD}} / m_{b}$ and $\Lambda_{\mathrm{QCD}} / E$, where $E$ is the energy of the light meson. Neglecting corrections of order $1 / m_{b}$ and $\alpha_{s}$, the seven a priori independent $B \rightarrow K^{*}$ form factors reduce to two universal form factors $\xi_{\perp}$ and $\xi_{\|}$[27, 28]. These relations can be strictly derived within the QCDf and SCET approach and are given in the appendix. Using those simplifications the spin amplitudes at leading order in $1 / m_{b}$ and $\alpha_{s}$ have a very simple form:

$$
A_{\perp L, R}=\sqrt{2} N m_{B}(1-\hat{s})\left[\left(\mathcal{C}_{9}^{(\text {eff })} \mp \mathcal{C}_{10}\right)+\frac{2 \hat{m}_{b}}{\hat{s}}\left(\mathcal{C}_{7}^{(\text {eff })}+\mathcal{C}_{7}^{\prime(\text { eff })}\right)\right] \xi_{\perp}\left(E_{K^{*}}\right)
$$




$$
\begin{aligned}
& A_{\| L, R}=-\sqrt{2} N m_{B}(1-\hat{s})\left[\left(\mathcal{C}_{9}^{(\mathrm{eff})} \mp \mathcal{C}_{10}\right)+\frac{2 \hat{m}_{b}}{\hat{s}}\left(\mathcal{C}_{7}^{(\mathrm{eff})}-\mathcal{C}_{7}^{\prime}(\mathrm{eff})\right] \xi_{\perp}\left(E_{K^{*}}\right),\right. \\
& A_{0 L, R}=-\frac{N m_{B}}{2 \hat{m}_{K^{*}} \sqrt{\hat{s}}}(1-\hat{s})^{2}\left[\left(\mathcal{C}_{9}^{(\mathrm{eff})} \mp \mathcal{C}_{10}\right)+2 \hat{m}_{b}\left(\mathcal{C}_{7}^{(\mathrm{eff})}-\mathcal{C}_{7}^{\prime(\text { eff })}\right)\right] \xi_{\|}\left(E_{K^{*}}\right),
\end{aligned}
$$

with $\hat{s}=q^{2} / m_{B}^{2}, \hat{m}_{i}=m_{i} / m_{B}$. Here we neglected terms of $O\left(\hat{m}_{K^{*}}^{2}\right)$.

Some remarks are in order:

- The theoretical simplifications are restricted to the kinematic region in which the energy of the $K^{*}$ is of the order of the heavy quark mass, i.e. $q^{2} \ll m_{B}^{2}$. Moreover, the influences of very light resonances below $1 \mathrm{GeV}^{2}$ question the QCD factorization results in that region. Thus, we will confine our analysis of all observables to the dilepton mass in the range, $1 \mathrm{GeV}^{2} \leqslant q^{2} \leqslant 6 \mathrm{GeV}^{2}$.

- Within the SM, we recover the naive quark-model prediction of $A_{\perp}=-A_{\|}$[29, 30] in the $m_{B} \rightarrow \infty$ and $E_{K^{*}} \rightarrow \infty$ limit (equivalently $\hat{m}_{K^{*}}^{2} \rightarrow 0$ ). In this case, the $s$ quark is produced in helicity $-1 / 2$ by weak interactions in the limit $m_{s} \rightarrow 0$, which is not affected by strong interactions in the massless case 31. Thus, the strange quark combines with a light quark to form a $K^{*}$ with helicity either -1 or 0 but not +1 . Consequently, the SM predicts at quark level $H_{+1}=0$, and hence $A_{\perp}=-A_{\|}$ [cf. Eq. (3.1)], which is revealed as $\left|H_{-1}\right| \gg\left|H_{+1}\right|$ (or $A_{\perp} \approx-A_{\|}$) at the hadron level.

- As noted in Ref. [19], the contributions of the chirality-flipped operators $\mathcal{O}_{9,10}^{\prime}=$ $\mathcal{O}_{9,10}\left(P_{L} \rightarrow P_{R}\right)$ can be included in the above amplitudes by the replacements $\mathcal{C}_{9,10}^{\text {(eff) }} \rightarrow$ $\mathcal{C}_{9,10}^{(\text {eff })}+\mathcal{C}_{9,10}^{\prime(\text { eff })}$ in Eq. (3.7), $\mathcal{C}_{9,10}^{(\text {eff })} \rightarrow \mathcal{C}_{9,10}^{(\text {eff })}-\mathcal{C}_{9,10}^{\prime(\text { eff })}$ in Eqs. (3.8) and (3.9). However, they play a sub-dominant role in our NP analysis presented here.

- The symmetry breaking corrections of order $\alpha_{s}$ can be calculated in the QCDf/SCET approach. Those NLO corrections are included in our numerical analysis following Ref. 117]. The corresponding formulae for the case $\mathcal{C}_{7}^{\prime(\text { eff })} \neq 0$ are given in Appendix $\mathrm{C}$.

- In general we have no means to calculate $\Lambda / m_{b}$ corrections to the QCDf amplitudes so they are treated as unknown corrections. This leads to a large uncertainty of theoretical predictions based on the QCDf/SCET approach. However, in specific examples one can combine QCDf/SCET results with calculations based on the QCD sum rule approach in order to estimate the leading power corrections.

To take into account the present situation, we introduce a set of extra parameters, one for each spin amplitude, to explore what the effect of a possible $\Lambda / m_{b}$ correction could be:

$$
A_{\perp, \|, 0}=A_{\perp, \|, 0}^{0}\left(1+c_{\perp, \|, 0}\right)
$$

where the ' 0 ' superscript stands for the QCD NLO Factorization amplitude and $c_{\perp, \|, 0}$ are taken to vary in a range $\pm 10 \%$ which corresponds to a naive dimensional estimate. For each observable we look at, each of the amplitudes were varied in turn leaving 
the others at their central value. All the variations were then added in quadrature. Furthermore, we also give our final predictions taking into account further improvements on the power corrections and varying the independent parameters in a less conservative range of $\pm 5 \%$.

\section{Theoretically clean observables}

\subsection{General criteria}

We recall again that 2 of the 11 measurable distribution functions $a, b, \ldots, m$ of the differential decay distribution in the limit $m_{\ell}^{2} \ll q^{2}$, defined in Eq. 2.3, include redundant information due to the relations $a=3 c$ and $b=-d$. So in principle there are 9 independent observables. However, the dependence of those functions on the six complex theoretical

spin amplitudes, $A_{\perp L / R}, A_{\| L / R}$ and $A_{0 L / R}$, is special. By inspection one finds that the distribution functions are invariant under the following three independent symmetry transformations of the spin amplitudes: global phase transformation of the $L$-amplitudes

$$
A_{\perp L}^{\prime}=e^{i \phi_{L}} A_{\perp L}, \quad A_{\| L}^{\prime}=e^{i \phi_{L}} A_{\| L}, \quad A_{0 L}^{\prime}=e^{i \phi_{L}} A_{0 L},
$$

global phase transformation of the $R$-amplitudes

$$
A_{\perp R}^{\prime}=e^{i \phi_{R}} A_{\perp R}, \quad A_{\| R}^{\prime}=e^{i \phi_{R}} A_{\| R}, \quad A_{0 R}^{\prime}=e^{i \phi_{R}} A_{0 R},
$$

and a continuous $L \leftrightarrow R$ rotation

$$
\begin{aligned}
A_{\perp L}^{\prime} & =+\cos \theta A_{\perp L}+\sin \theta A_{\perp R}^{*} \\
A_{\perp R}^{\prime} & =-\sin \theta A_{\perp L}^{*}+\cos \theta A_{\perp R} \\
A_{0 L}^{\prime} & =+\cos \theta A_{0 L}-\sin \theta A_{0 R}^{*} \\
A_{0 R}^{\prime} & =+\sin \theta A_{0 L}^{*}+\cos \theta A_{0 R} \\
A_{\| L}^{\prime} & =+\cos \theta A_{\| L}-\sin \theta A_{\| R}^{*} \\
A_{\| R}^{\prime} & =+\sin \theta A_{\| L}^{*}+\cos \theta A_{\| R} .
\end{aligned}
$$

Normally, there is the freedom to pick a single global phase, but as $L$ and $R$ amplitudes do not interfere here, two phases can be chosen arbitrarily as reflected in the first two transformations. The third symmetry reflects that an average is made over the spin amplitudes to obtain the angular distribution. So it is clear that only 9 out of the 12 parameters arising from the 6 complex amplitudes are independent which fits exactly with the 9 independent measurable distribution functions.

A consequence of the three symmetries is that any observable based on the differential decay distribution has also to be invariant under the same symmetry transformations.

Besides the mandatory criterion above there are further criteria required for an interesting observable:

Simplicity: A simple functional dependence on the 9 independent measurable distribution functions; at best it should depend only from one or two in the numerator and denominator of an asymmetry. 
Cleanliness: At leading order in $\Lambda / m_{b}$ and in $\alpha_{s}$ the observable should be independent of any form factor, at best for all $q^{2}$. Also the influence of symmetry-breaking corrections at order $\alpha_{s}$ and at order $\Lambda / m_{b}$ should be minimal.

Sensitivity: The sensitivity to the $\mathcal{C}_{7}^{\prime}{ }^{(\text {eff })}$ Wilson coefficient representing NP with another chirality than in the SM should be maximal.

Precision: The experimental precision obtainable should be good enough to distinguish different NP models.

In the limit where the $\bar{K}^{* 0}$ meson has a large energy, only two independent form factors occur in $A_{0 L / R}$ and in $A_{\perp L / R}$ and $A_{\| L / R}$. Clearly, any ratio of two of the nine measurable distribution functions proportional to the same form factor fulfil the criterion of symmetry, simplicity, and theoretical cleanliness up to $\Lambda / m_{b}$ and $\alpha_{s}$ corrections. However, the third criterion, a sensitivity to a special kind of NP and the subsequent requirement of experimental precision, singles out particular combinations. In this paper we focus on new right-handed currents. Other NP sensitivities may single out other observables as will be analysed in a forthcoming paper 32].

\subsection{Observables}

There are some proposals for theoretical clean observables already in the literature which we should briefly discuss in view of the above criteria:

- The forward backward asymmetry is the most popular quantity in the $\bar{B}_{d} \rightarrow \bar{K}^{* 0} \mu^{+} \mu^{-}$ decay [33]. In terms of the $\bar{K}^{* 0}$ spin amplitudes it can be written as [17, 34]

$$
A_{\mathrm{FB}}=\frac{3}{2} \frac{\operatorname{Re}\left(A_{\| L} A_{\perp L}^{*}\right)-\operatorname{Re}\left(A_{\| R} A_{\perp R}^{*}\right)}{\left|A_{0}\right|^{2}+\left|A_{\|}\right|^{2}+\left|A_{\perp}\right|^{2}}
$$

where

$$
A_{i} A_{j}^{*} \equiv A_{i L}\left(q^{2}\right) A_{j L}^{*}\left(q^{2}\right)+A_{i R}\left(q^{2}\right) A_{j R}^{*}\left(q^{2}\right) \quad(i, j=0, \|, \perp) .
$$

While the criteria of symmetry and simplicity are fulfilled, the form factors cancel out only at the specific value of $q^{2}$ where $A_{\mathrm{FB}}=0$. Thus the measurement provide only a single clean number, the zero crossing point, rather than a theoretically clean distribution.

- The fractions of the $\bar{K}^{* 0}$ polarisation

$$
\begin{aligned}
& F_{L}\left(q^{2}\right)=\frac{\left|A_{0}\right|^{2}}{\left|A_{0}\right|^{2}+\left|A_{\|}\right|^{2}+\left|A_{\perp}\right|^{2}}, \\
& F_{T}\left(q^{2}\right)=1-F_{L}\left(q^{2}\right)=\frac{\left|A_{\perp}\right|^{2}+\left|A_{\|}\right|^{2}}{\left|A_{0}\right|^{2}+\left|A_{\|}\right|^{2}+\left|A_{\perp}\right|^{2}},
\end{aligned}
$$

and the $K^{*}$ polarisation parameter

$$
\alpha_{K^{*}}\left(q^{2}\right)=\frac{2 F_{L}}{F_{T}}-1=\frac{2\left|A_{0}\right|^{2}}{\left|A_{\|}\right|^{2}+\left|A_{\perp}\right|^{2}}-1
$$


All fulfil the criteria of symmetry and simplicity, but the form factors do not cancel in the LO approximation; thus, suffering from larger hadronic uncertainties. The fraction of the $\bar{K}^{*}$ polarisation can be measured from the angular projections alone and the first experimental measurements of $F_{L}$ with limited accuracy are available 8 , 9.

- Defining the helicity distributions $\Gamma_{ \pm}=\left|H_{ \pm 1}^{L}\right|^{2}+\left|H_{ \pm 1}^{R}\right|^{2}$ one can construct [23]

$$
A_{T}^{(1)}=\frac{\Gamma_{-}-\Gamma_{+}}{\Gamma_{-}+\Gamma_{+}}=\frac{-2 \operatorname{Re}\left(A_{\|} A_{\perp}^{*}\right)}{\left|A_{\perp}\right|^{2}+\left|A_{\|}\right|^{2}} .
$$

It has been shown 19, 20] that this quantity has adequate cleanliness and is is very sensitive to right-handed currents, making an ideal observable if just these two criteria were sufficient. However, the quantity $A_{T}^{(1)}$ does not fulfil the most important criterion of symmetry. The important consequences out of this observation are briefly discussed in the next subsection.

- The other transversity amplitude, first proposed in [19], is defined as

$$
A_{T}^{(2)}=\frac{\left|A_{\perp}\right|^{2}-\left|A_{\|}\right|^{2}}{\left|A_{\perp}\right|^{2}+\left|A_{\|}\right|^{2}} .
$$

It obviously fulfils all three criteria of symmetry, simplicity and theoretical cleanliness. It is also rather sensitivity to $\mathcal{C}_{7}^{\prime}$ (eff) as one can see by inspection of the LO formulae of the $\bar{K}^{* 0}$ amplitudes in Eqs. (3.7 3.9); in this approximation it is directly proportional to $\mathcal{C}_{7}^{\prime(\text { eff })}$, thus vanishes in the SM.

By inspection of the formulae of the $K^{*}$ spin amplitudes in terms of the Wilson coefficients and the SCET form factors at the LO approximation, Eqs. (3.7 3.9), one is led to some new observables which fulfil the first three criteria and have an enhanced sensitivity to $\mathcal{C}_{7}^{\prime}{ }^{\prime}$ eff . They are defined as

$$
A_{T}^{(3)}=\frac{\left|A_{0 L} A_{\| L}^{*}+A_{0 R}^{*} A_{\| R}\right|}{\sqrt{\left|A_{0}\right|^{2}\left|A_{\perp}\right|^{2}}},
$$

and

$$
A_{T}^{(4)}=\frac{\left|A_{0 L} A_{\perp L}^{*}-A_{0 R}^{*} A_{\perp R}\right|}{\left|A_{0 L}^{*} A_{\| L}+A_{0 R} A_{\| R}^{*}\right|}
$$

One could also consider the real and imaginary parts of $A_{T}^{(3)}$.

There are no further independent quantities which fulfil the criteria we have set out. However, when we will consider NP sensitivities beyond $\mathcal{C}_{7}^{\prime}{ }^{(\text {eff })}$ further observables may be singled out 32.

\subsection{The problem with $A_{T}^{(1)}$}

Contrary to the case of $A_{T}^{(i)}$ with $i=2,3,4$, it is not possible to extract $A_{T}^{(1)}$ from the full angular distribution. This is a direct consequence of the fact that the quantity $A_{T}^{(1)}$ is 
not invariant under the symmetry (4.3) of the distribution function (2.1) which represent the complete set of observables in the case spins of the final states are summed up. Let us elaborate further on this surprising observation; it seems practically not possible to measure the helicity of the final states on a event-by-event basis. At the forthcoming $\mathrm{LHC} b$ experiment for example one only measures the charge, the three-momentum of the final state particles and its nature through different types of particle identification. So one has the four-momentum for each particle and its charge. The situation does not look different for the present $B$ factories and their future upgrades. While the $e^{+} e^{-}$environment is much simpler there is still no practical way to measure the spin of the muons on an event-by-event basis. We should emphasise that this is a practical and not a conceptual problem; in a gedanken experiment where the helicity of the individual final state leptons are measured, it would indeed be possible to measure $A_{T}^{(1)}$. So while $A_{T}^{(1)}$ is in principle a good observable, we cannot see any way it can be measured at either $\mathrm{LHC} b$ or at a $\operatorname{Super}-B$ factory with electrons or muons in the final state.

\section{Method to calculate experimental sensitivity}

In this section we explain how to investigate the sensitivity to the angular observables presented in Sec. 1 using a toy Monte Carlo model. We estimate the statistical uncertainty on all observables with statistics corresponding to 5 years of nominal running at $\mathrm{LHC} b$ $\left(10 \mathrm{fb}^{-1}\right)$ and comment on the experimental prospects for a measurement at the end of an upgrade to $\mathrm{LHC} b\left(100 \mathrm{fb}^{-1}\right)$. For the estimates here we are only considering the final state with muons.

\section{$5.1 \bar{B}_{d} \rightarrow \bar{K}^{* 0} \ell^{+} \ell^{-}$decay model}

The angles $\theta_{l}, \theta_{K}$ and $\phi$, as well as the $q^{2}$ of the lepton pair can be measured with small uncertainty and no experimental resolution effects need to be considered. A toy Monte Carlo model of the decay was created using Eq. (2.1) as a probability density function (PDF) normalised to the width,

$$
\int_{q_{\min }^{2}}^{q_{\max }^{2}} \frac{d \Gamma}{d q^{2}} d q^{2} .
$$

It is parameterised in terms of the real and imaginary parts of the spin amplitudes where each of these amplitudes is $q^{2}$ dependent. A simple approach, where the data is divided into regions of $q^{2}$ and the spin amplitudes determined within these, will not work; the coefficients in front of the different angular components as seen in Eq. (2.1) depend in a non-linear way on the spin amplitudes meaning that the angular distribution after integration over a bin in $q^{2}$ cannot be expressed in terms of Eq. (2.1) with some $q^{2}$-averaged spin amplitudes. Instead an approach is used where the $q^{2}$ dependence of each of the spin amplitudes is parameterised as a function of $q^{2}$.

A special choice of the symmetry transformations described in Sec. 1 can be used to reduce the number of parameters. Here we use the first two symmetry transformations Eqs. (4.1) and (4.2) to get rid of the two phases in $A_{0 R}$ and $A_{0 L}$. Then the third transformation Eq. (4.3) is used with $\theta=\arctan \left(-A_{0 R} / A_{0 L}\right)$ leading to $A_{0 L}$ being real and 
$A_{0 R}=0$ thus disappearing completely from the parametrisation. At a given value of $q^{2}$ we are thus left with 9 parameters corresponding to the real and imaginary components of $A_{\| L, R}$ and $A_{\perp L, R}$ and the real component of $A_{0 L}$. We now parameterise each of these spin amplitudes as a $2^{\text {nd }}$ order polynomial. Through the polynomial ansatz we are introducing a weak model dependence; we checked that the error introduced by this was significantly smaller than the corresponding experimental errors across the squared dimuon mass range $1 \mathrm{GeV}^{2}<q^{2}<6 \mathrm{GeV}^{2}$. To describe the full $q^{2}$ and angular dependence of the decay we thus need 27 parameters. As a final step we recognise that an absolute measurement of the total width is difficult to obtain in a hadronic environment such as $\mathrm{LHC} b$ and fix the value of $A_{0 L}$ to 1 at a reference value of $q^{2}$ thus reducing the number of free parameters to 26 . This last step has no influence on the experimental determination of any of the observables discussed in this paper as they are all formed as ratios where the total width cancels out. While no longer sensitive to the absolute width we are still sensitive to the shape of the differential width as a function of $q^{2}$.

We follow the resolution, yield and background numbers in 10 to construct a model that includes a realistic level of background. The signal is assumed to have a Gaussian distribution in $m_{B}$ with a width of $14 \mathrm{MeV}$ in a window of $m_{B} \pm 50 \mathrm{MeV}$ and a Breit-Wigner in $m_{K \pi}$ with width $48 \mathrm{MeV}$ in a window of $m_{K^{* 0}} \pm 100 \mathrm{MeV}$. A simplified background model is included; it is flat in all angles, effectively treating all background as combinatorial, but follows the $q^{2}$ distribution of the signal. Acceptance and $C P$ violation effects are neglected allowing us to treat $\bar{B}_{d} \rightarrow \bar{K}^{* 0} \mu^{+} \mu^{-}$and its charge conjugate simultaneously. We do not include any contributions from non-resonant $\bar{B}_{d} \rightarrow K^{-} \pi^{+} \mu^{+} \mu^{-}$.

Using the toy Monte Carlo model, a dataset for the observables $\theta_{l}, \theta_{K}, \phi$ and $q^{2}$ can be generated with the calculated values of the spin amplitudes as input without making use of the polynomial ansatz. Physics beyond the SM can be included in a straightforward way by providing the relevant spin amplitudes. Using the yield and background estimates from [10] and assuming a flat efficiency for the signal as a function of $q^{2}$ we use on average 4032 signal events and 1168 background events in the $q^{2}$ interval from $4 m_{\mu}^{2}$ to $9 \mathrm{GeV}^{2}$ in a dataset of $2 \mathrm{fb}^{-1}$. These are scaled lineally in order to obtain $10 \mathrm{fb}^{-1}$ and $100 \mathrm{fb}^{-1}$ yield estimates. For each dataset we generate, the signal and background numbers are varied according to Poisson statistics.

The purpose of the toy Monte Carlo model is to enable us to illustrate the methodology of this approach and be able to make precise statements on the relative performance of a full angular fit compared to just looking at projections. Accurate estimates of the resolution in each parameter will only be possible with a complete detector simulation and with a complete understanding of the actual detector performance following the first data.

\subsection{Full angular fit}

With the model above we can generate an ensemble of experiments corresponding to a given integrated luminosity. In each of these experiments we can use a general minimiser to find the spin value parametrisation that best corresponds to the data. Each fit has in total 27 parameters; 26 from the signal described above and a single parameter to describe the level of the simplified background model. From the ensemble of experiments, estimates 


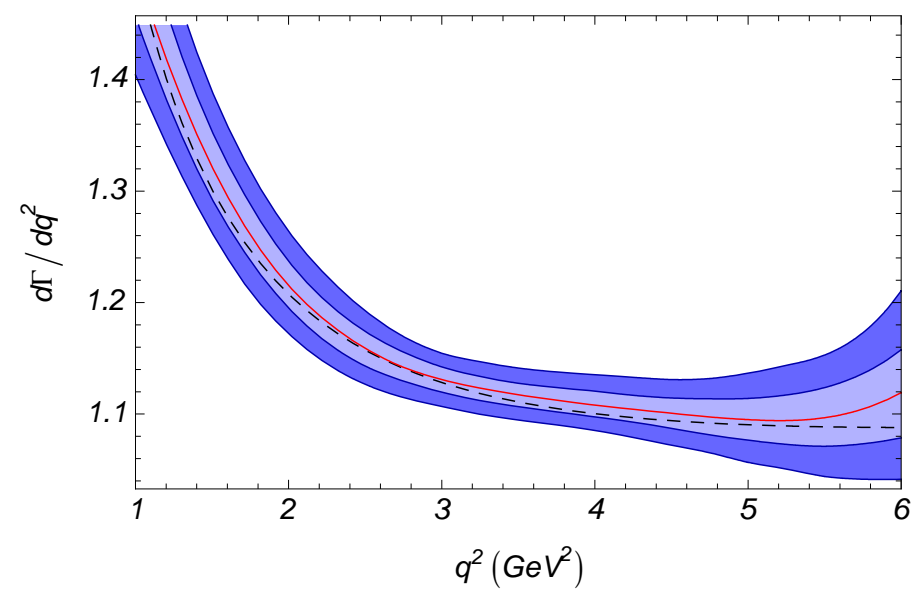

Figure 1: The experimental sensitivity to $\frac{d \Gamma}{d q^{2}}$ with the SM as input. The inner and outer bands correspond to $1 \sigma$ and $2 \sigma$ experimental errors with statistics corresponding to a $10 \mathrm{fb}^{-1}$ dataset from $\mathrm{LHC} b$. The black dashed line is the theoretical input and the red solid line the central value of the ensemble experiments.

of the experimental uncertainties can be made and any biases introduced can be studied. For each dataset, the extracted spin amplitude components were used to calculate the value of each angular observable as a function of $q^{2}$. In total we created an ensemble of 1000 experiments and will thus at a given value of $q^{2}$ get 1000 different determinations of a given observable. By looking at the point where $33 \%$ and $47.5 \%$ of results lie within either side of the median of the results we can form asymmetric $1 \sigma$ and $2 \sigma$ errors. Connecting these at different $q^{2}$ values gives us $1 \sigma$ and $2 \sigma$ bands for the experimental errors on the observable. An illustration of the method in Fig. 1 shows the experimental sensitivity to the width distribution relative to the normalisation point which was arbitrarily chosen as $3.5 \mathrm{GeV}^{2}$. The inner and outer bands correspond to $1 \sigma$ and $2 \sigma$ experimental errors with statistics corresponding to a $10 \mathrm{fb}^{-1}$ dataset from $\mathrm{LHC} b$. The dashed line is the theoretical input and the red line the central value of the ensemble experiments. The difference between these two lines is caused by limitations imposed by the second order polynomial assumption. As it is is well inside the $1 \sigma$ band this is not problem.

The experimental sensitivity to the observables introduced in Sec. 4.2 will be presented in Sec. 6 within the phenomenological analysis to allow for an easy comparison of experimental and theoretical errors.

\subsection{Comparisons with fits to projections}

The full angular fit gives access to angular observables not accessible in other ways. However, $A_{\mathrm{FB}}, A_{T}^{(2)}, F_{\mathrm{L}}$ and $A_{\mathrm{im}}{ }^{1}$ can be extracted from distributions in just a single angle

\footnotetext{
${ }^{1} A_{\mathrm{im}}$ is defined as $A_{\mathrm{im}}=\frac{\operatorname{Im}\left(A_{\perp L} A_{\| L}^{*}\right)+\operatorname{Im}\left(A_{\perp R} A_{\| R}^{*}\right)}{\left|A_{0}\right|^{2}+\left|A_{\perp}\right|^{2}+\left|A_{\|}\right|^{2}}$ and is included for completeness here. It is not of importance for the measurement of right handed currents.
} 
after integration over the other 2 in Eq. (2.1):

$$
\begin{aligned}
\frac{d \Gamma^{\prime}}{d \phi} & =\frac{\Gamma^{\prime}}{2 \pi}\left(1+\frac{1}{2}\left(1-F_{\mathrm{L}}\right) A_{T}^{(2)} \cos 2 \phi+A_{\mathrm{im}} \sin 2 \phi\right) \\
\frac{d \Gamma^{\prime}}{d \theta_{l}} & =\Gamma^{\prime}\left(\frac{3}{4} F_{\mathrm{L}} \sin ^{2} \theta_{l}+\frac{3}{8}\left(1-F_{\mathrm{L}}\right)\left(1+\cos ^{2} \theta_{l}\right)+A_{\mathrm{FB}} \cos \theta_{l}\right) \sin \theta_{l}, \\
\frac{d \Gamma^{\prime}}{d \theta_{K}} & =\frac{3 \Gamma^{\prime}}{4} \sin \theta_{K}\left(2 F_{\mathrm{L}} \cos ^{2} \theta_{K}+\left(1-F_{\mathrm{L}}\right) \sin ^{2} \theta_{K}\right),
\end{aligned}
$$

where $\Gamma^{\prime}=b+4 c$. This method was investigated for LHC $b$ in [12]. The observables appear linearly in the expressions so the fits can be performed on data binned in $q^{2}$. The value extracted from these fits is then a $\frac{d \Gamma}{d q^{2}}$ weighted average of each parameter.

The full angular model described in Sec. 5.2 was used to generate data sets which were then fit simultaneously using the distributions in Eq. (5.2). The treatment of background and the $m_{B, K \pi}$ distributions were the same as in the full angular model. For a direct comparison between this method and the full angular fit, the $q^{2}$ dependent values of the observables were averaged using a weighted mean,

$$
A_{T}^{(i)}=\frac{\int_{q_{\min }^{2}}^{q_{\max }^{2}} \frac{d \Gamma}{d q^{2}} A_{T}^{(i)}\left(q^{2}\right)}{\int_{q_{\min }^{2}}^{q_{\max }^{2}} \frac{d \Gamma}{d q^{2}}} .
$$

The central values produced for the full angular approach in this case show some small biases due to the breakdown of the polynomial ansatz at the edges of the $q^{2}$ distribution, however this is still well below the statistical error expected with $10 \mathrm{fb}^{-1}$ of data from $\mathrm{LHC} b$. The power of the full angular fit is striking for $A_{T}^{(2)}$ where the resolution is above a factor 2 better compared to fitting the projections. This can easily be understood in terms of the $\left(1-F_{\mathrm{L}}\right)$ factor in Eq. (5.2a), where $F_{\mathrm{L}}$ is large in the SM.

For all the observables where a comparison can be made, we see that the full angular fit provides improvements in the resolution of between $15 \%$ and $60 \%$.

In the full angular fit we can calculate the position of the zero crossing for the forwardbackward asymmetry, $q_{0}^{2}$. We illustrate the distribution of results obtained from the ensemble of datasets in Fig. 2 2 where a resolution, assuming the SM as input, of $0.17 \mathrm{GeV}^{2}$ is obtained. Alternatively we can perform the simpler task of binning the data in $1 \mathrm{GeV}^{2}$ bins and then in each bin perform simultaneous fits to the three angular projections. The value of $A_{\mathrm{FB}}$ is extracted by performing a straight line fit in the range $2-6 \mathrm{GeV}^{2}$ to the $A_{\mathrm{FB}}$ values found in each $q^{2}$ bin. This gives us, with exactly the same assumptions for how background and acceptance are treated, a resolution of $0.24 \mathrm{GeV}^{2}$. So also in this case we see an improvement of $30 \%$ in the statistical power by performing a full angular fit.

The comparisons made here demonstrate that there is significant advantage in performing the full angular fit once the data sets are large enough. Using the simplified model described here it is possible to use this approach even with a smaller $2 \mathrm{fb}^{-1}$ data set. In reality, detector effects not accounted for such as angular acceptance will complicate the process and a proper full angular analysis may not be possible with data sets this small. However, we have shown that with the signal and background statistics at LHC $b$ a full angular analysis is possible once the detector effects have been properly understood. 


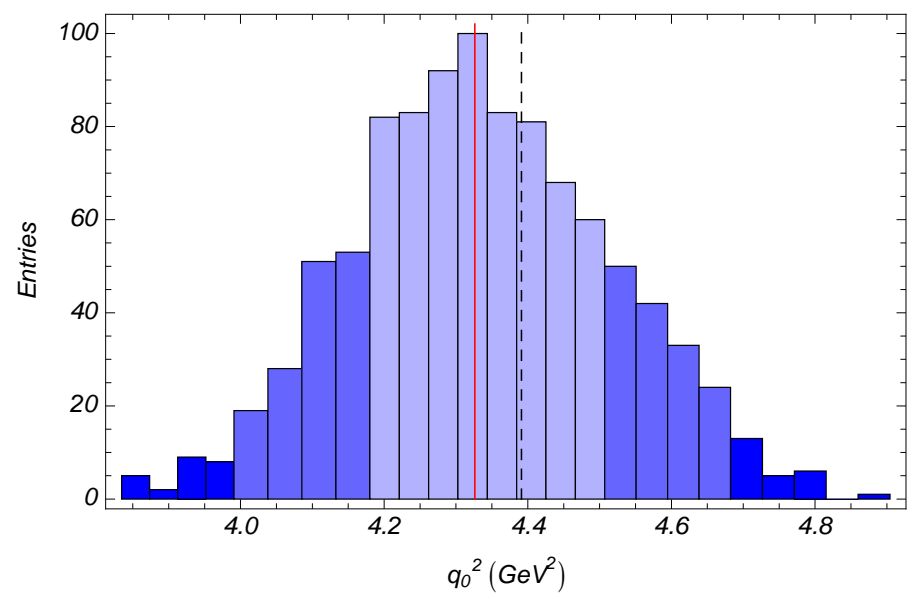

Figure 2: The distribution in the determination of the zero crossing of $A_{\mathrm{FB}}$ from an ensemble of datasets created from the toy Monte Carlo model with statistics corresponding to $10 \mathrm{fb}^{-1}$ at $\mathrm{LHC} b$. The edge of the inner (light blue) and middle (medium blue) regions correspond to $1 \sigma$ and $2 \sigma$ experimental errors. The solid (red) line is the median of the fitted values and the dashed (black) line is the input value from the SM theory predictions. From the figure we see a resolution of $0.17 \mathrm{GeV}^{2}$.

\section{Phenomenological analysis}

In this section we present our phenomenological analysis of the old and new observables in the SM and in extensions of the SM with new right-handed currents. The latter can be done in a model independent way by introducing the chiral partners of the SM Wilson coefficients $\mathcal{C}_{7}^{(\text {eff })}, \mathcal{C}_{9}^{(\text {eff })}$, and $\mathcal{C}_{10}{ }^{2}$. This general new-physics scenario can be realized for example via gluino-mediated FCNC in the general R-parity conserving MSSM.

\subsection{Preliminaries}

Our analysis is based on the numerical input as summarized in Table1. Regarding the form factor value we follow Ref. [35] and use the value fixed by experimental data. Moreover, we introduce four representative benchmark points of supersymmetry with non-minimal flavour violation in the down squark sector which were already used in Ref. [20]. The most important flavour diagonal parameters are fixed as follows: $\tan \beta=5, \mu=M_{1}=M_{2}=$ $M_{H^{+}}=m_{\tilde{u}_{R}}=1 \mathrm{TeV}$. Note that we choose a low value for $\tan \beta$; this shows that we do not need to rely on a large-tan $\beta$ to see an effect, and ensures automatic fulfilment of the constraint coming from $B_{s} \rightarrow \mu^{+} \mu^{-}$. Furthermore, we make the assumption that all the entries in $m_{u, L R}^{2}$ and $m_{d, L R}^{2}$ vanish, with the exception of the one that corresponds to $\left(\delta_{L R}^{d}\right)_{32}$. The remaining parameters of the four benchmark points correspond to two different scenarios and are fixed as follows ${ }^{3}$ :

\footnotetext{
${ }^{2}$ We note here that the impact of $\mathcal{C}_{9}^{(\text {eff })}$ and $\mathcal{C}_{10}$ and their chiral partners is rather small compared with $\mathcal{C}_{7}^{\prime}{ }^{(\text {eff })}$ in the low- $q^{2}$ region, due to the $2 \hat{m}_{b} / \hat{s}$ factor in the matrix element and the experimental constraints from the inclusive decay $B \rightarrow X_{s} \ell^{+} \ell^{-}$.

${ }^{3}$ We follow here the conventions of Ref. 36.
} 


\begin{tabular}{|ll|ll|}
\hline \hline$m_{B}$ & $5.27950 \pm 0.00033 \mathrm{GeV}$ & $\lambda$ & $0.2262 \pm 0.0014$ \\
$m_{K}$ & $0.896 \pm 0.040 \mathrm{GeV}$ & $A$ & $0.815 \pm 0.013$ \\
$M_{W}$ & $80.403 \pm 0.029 \mathrm{GeV}$ & $\bar{\rho}$ & $0.235 \pm 0.031$ \\
$M_{Z}$ & $91.1876 \pm 0.0021 \mathrm{GeV}$ & $\bar{\eta}$ & $0.349 \pm 0.020$ \\
\hline$\hat{m}_{t}\left(\hat{m}_{t}\right)$ & $167 \pm 5 \mathrm{GeV}$ & $\Lambda_{\mathrm{QCD}}^{\left(n_{f}=5\right)}$ & $220 \pm 40 \mathrm{MeV}$ \\
$m_{b, \mathrm{PS}}(2 \mathrm{GeV})$ & $4.6 \pm 0.1 \mathrm{GeV}$ & $\alpha_{s}\left(M_{Z}\right)$ & $0.1176 \pm 0.0002$ \\
$m_{c}$ & $1.5 \pm 0.2 \mathrm{GeV}$ & $\alpha_{\mathrm{em}}$ & $1 / 137$ \\
\hline$f_{B}$ & $200 \pm 30 \mathrm{MeV}$ & $a_{1}\left(K^{*}\right)_{\perp, \|}$ & $0.10 \pm 0.07$ \\
$f_{K^{*}, \perp}$ & $175 \pm 25 \mathrm{MeV}$ & $a_{2}\left(K^{*}\right)_{\perp}$ & $0.13 \pm 0.08$ \\
$f_{K^{*}, \|}$ & $217 \pm 5 \mathrm{MeV}$ & $a_{2}\left(K^{*}\right)_{\|}$ & $0.09 \pm 0.05$ \\
\hline$m_{B} \xi_{K^{*}, \|}(0) /\left(2 m_{K^{*}}\right)$ & $0.47 \pm 0.09$ & $\lambda_{B,+}(1.5 \mathrm{GeV})$ & $0.485 \pm 0.115 \mathrm{GeV}$ \\
$\xi_{K^{*}, \perp}(0)$ & $0.26 \pm 0.02$ & & \\
\hline \hline
\end{tabular}

Table 1: Summary of input parameters and estimated uncertainties.

- Scenario A: $m_{\tilde{g}}=1 \mathrm{TeV}$ and $m_{\tilde{d}} \in[200,1000] \mathrm{GeV}$. The only non-zero mass insertion is varied between $-0.1 \leq\left(\delta_{L R}^{d}\right)_{32} \leq 0.1$. For all parameter sets the compatibility with other $B$ physics constraints, the electroweak constraints, constraints from particle searches, and also with the vacuum stability bounds is verified [20]. The curves denoted by (a) and (b) correspond respectively to $m_{\tilde{g}} / m_{\tilde{d}}=2.5,\left(\delta_{L R}^{d}\right)_{32}=0.016$ and $m_{\tilde{g}} / m_{\tilde{d}}=4,\left(\delta_{L R}^{d}\right)_{32}=0.036$. We will refer to this case as the large-gluino and positive mass insertion scenario. In terms of the effective Wilson coefficients at $m_{b}$, model (a) corresponds to $\left(\mathcal{C}_{7}^{(\text {eff })}, \mathcal{C}_{7}^{\prime}{ }^{(\text {eff })}\right)=(-0.32,0.16)$ and (b) to $(-0.32,0.24)$. This should be compared to the $\mathrm{SM}$ value of $\left(\mathcal{C}_{7}^{(\text {eff })}, \mathcal{C}_{7}^{\prime}(\right.$ eff $)=(-0.31,0.00)$.

- Scenario B: $m_{\tilde{d}}=1 \mathrm{TeV}$ and $m_{\tilde{g}} \in[200,800] \mathrm{GeV}$. The mass insertion is varied in the same range as Scenario A. The curves denoted by (c) and (d) correspond respectively to $m_{\tilde{g}} / m_{\tilde{d}}=0.7,\left(\delta_{L R}^{d}\right)_{32}=-0.004$ and $m_{\tilde{g}} / m_{\tilde{d}}=0.6,\left(\delta_{L R}^{d}\right)_{32}=$ -0.006 . We will refer to this case as the low-gluino mass (although large squark mass would be more appropriate) and negative mass insertion scenario. In this case the corresponding effective Wilson coefficients are $\left(\mathcal{C}_{7}^{\text {(eff) }}, \mathcal{C}_{7}^{\prime(\text { eff })}\right)=(-0.32,-0.08)$ for (c) and $(-0.32,-0.13)$ for $(d)$.

Notice that we have changed curve (c) with respect to Ref. 20] reducing its corresponding mass insertion to avoid any conflict with vacuum stability or colour breaking constraints [37].

Finally, we emphasize again that the validity of our theoretical predictions is restricted to the kinematic region in which the energy of the $K^{*}$ is of the order of the heavy quark mass. So we restrict our analysis to the low- $q^{2}$ region from $1 \mathrm{GeV}^{2}$ to $6 \mathrm{GeV}^{2}$. In the region below $1 \mathrm{GeV}^{2}$ the $\mathrm{QCDf} / \mathrm{SCET}$ results are questioned by the presence of very light resonances. 


\subsection{Results}

We present our results on the observables $A_{T}^{(2)}, A_{T}^{(3)}, A_{T}^{(4)}, A_{\mathrm{FB}}$ and $F_{L}$ in the Figs. 387 (for definitions see Sec. 四). For all the observables we plot the theoretical sensitivity on the left hand side of each Figure.

- The thin dark line is the central NLO result for the SM and the narrow inner dark (orange) band that surrounds it corresponds to the NLO SM uncertainties due to both input parameters and perturbative scale dependence. Light grey (green) bands are the estimated $\Lambda / m_{b} \pm 5 \%$ corrections for each spin amplitude (as given in Eq. 3.10) while darker grey (green) ones are the more conservative $\Lambda / m_{b} \pm 10 \%$ corrections. The curves labelled (a)-(d) correspond to the four different benchmark points in the MSSM introduced above.

- The experimental sensitivity for a dataset corresponding to $10 \mathrm{fb}^{-1}$ of $\mathrm{LHC} b$ data is given in each figure on the right hand side. Here the solid (red) line shows the median extracted from the fit to the ensemble of data and the dashed (black) line shows the theoretical input distribution. The inner and outer bands correspond to $1 \sigma$ and $2 \sigma$ experimental errors.
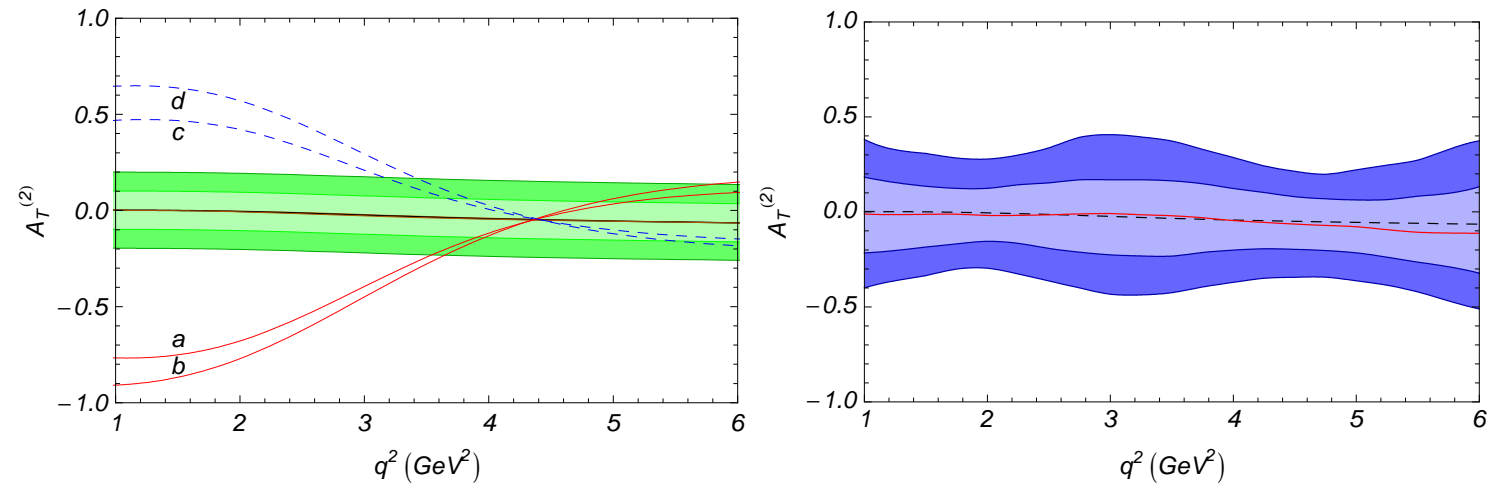

Figure 3: For $A_{T}^{(2)}$ we compare the theoretical errors (left) with the experimental errors (right) as a function of the squared dimuon mass. For the theory, the narrow inner dark (orange) bands correspond to the NLO result for the SM including all uncertainties (except for $\Lambda / m_{b}$ ) as explained in the text. Light grey (green) bands include the estimated $\Lambda / m_{b}$ uncertainty at a $\pm 5 \%$ level and the external dark grey (green) bands correspond to a $\pm 10 \%$ correction for each spin amplitude. The curves labelled (a)-(d) correspond to different SUSY scenarios as explained in the text. For the experimental aspects the inner and outer bands correspond to $1 \sigma$ and $2 \sigma$ statistical errors with a yield corresponding to a $10 \mathrm{fb}^{-1}$ dataset from $\mathrm{LHC}$.

Let us start with some concrete observations on the new observables $A_{T}^{(3)}$ and $A_{T}^{(4)}$. They offer sensitivity to the longitudinal spin amplitude $A_{0 L, R}$ in a controlled way compared to the old observables $F_{L}$ and $\alpha_{K}^{*}$ : the dependence on both the parallel and perpendicular soft form factors $\xi_{\|}(0)$ and $\xi_{\perp}(0)$ cancels at LO. A residual of this dependence may appear at NLO, but as shown in Figs. 1 and 5 , it is basically negligible. It is also remarkable 

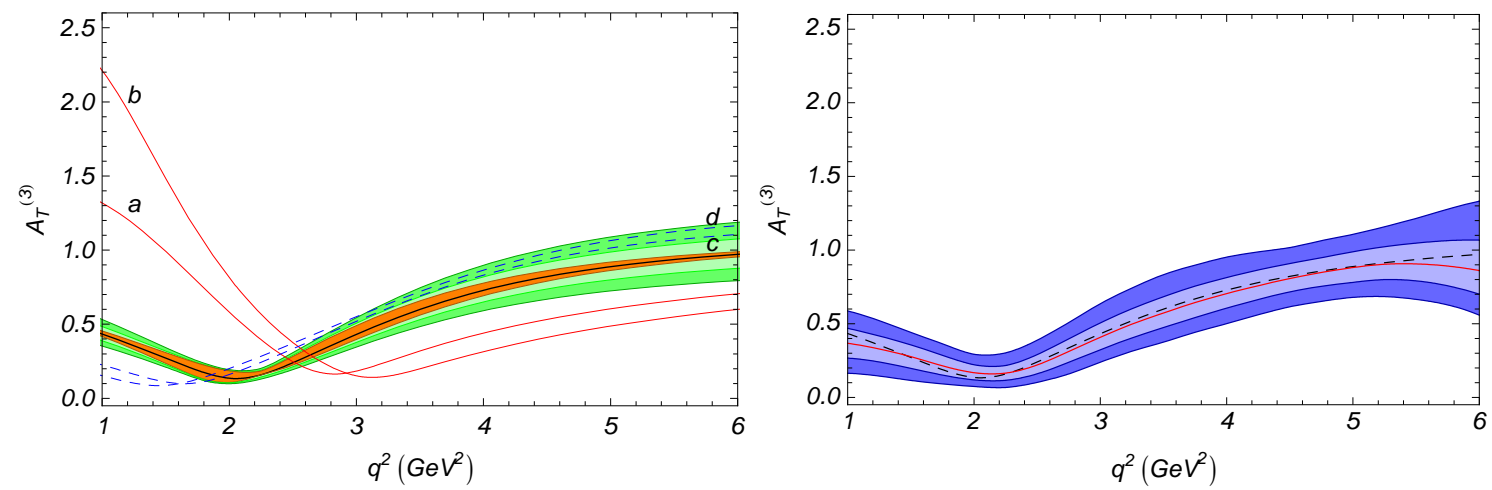

Figure 4: For the new observable $A_{T}^{(3)}$ we compare the theoretical errors (left) with the experimental errors (right). See the caption of Fig. 3 for details.
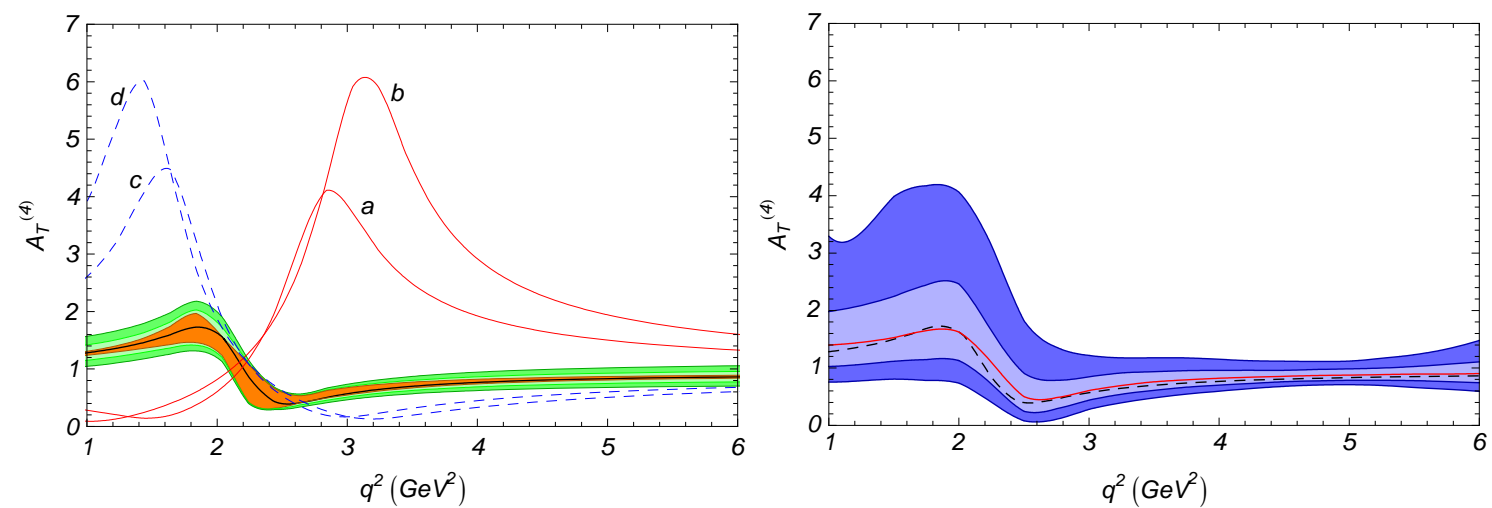

Figure 5: For the new observable $A_{T}^{(4)}$ we compare the theoretical errors (left) with the experimental errors (right). See the caption of Fig. 3 for details.

that for $A_{T}^{(3)}$ and $A_{T}^{(4)}$ at low $q^{2}$ the impact of this uncertainty is less important than the uncertainties due to input parameters and scale dependence.

The peaking structure in $A_{T}^{(4)}$ as a function of $q^{2}$ for the benchmark MSSM points is due to the different way $\mathcal{C}_{7}^{\prime}$ (eff) enters numerator and denominator; the numerator has a positive slope in the region of the peak, while the denominator has a minimum at the same point. If one uses the simplified L0 expressions from Eqs. 3.7 3.9 the denominator is exactly zero, generating an infinity at the point of the peak; however, once NLO QCDf is included the zero in the denominator is lifted and the result is a curve with a peak instead.

The new observables $A_{T}^{(3)}$ and $A_{T}^{(4)}$ also present a different sensitivity to $\mathcal{C}_{7}^{\prime}{ }^{(\text {eff })}$ via their dependence on $A_{0 L, R}$ compared with $A_{T}^{(2)}$. This may allow for a particularly interesting cross check of the sensitivity to this chirality flipped operator $\mathcal{O}_{7}^{\prime}$; for instance, new contributions coming from tensor scalars and pseudo-scalars will behave differently among the set of observables.

Another remarkable point that comes clear when comparing the set of clean observables 

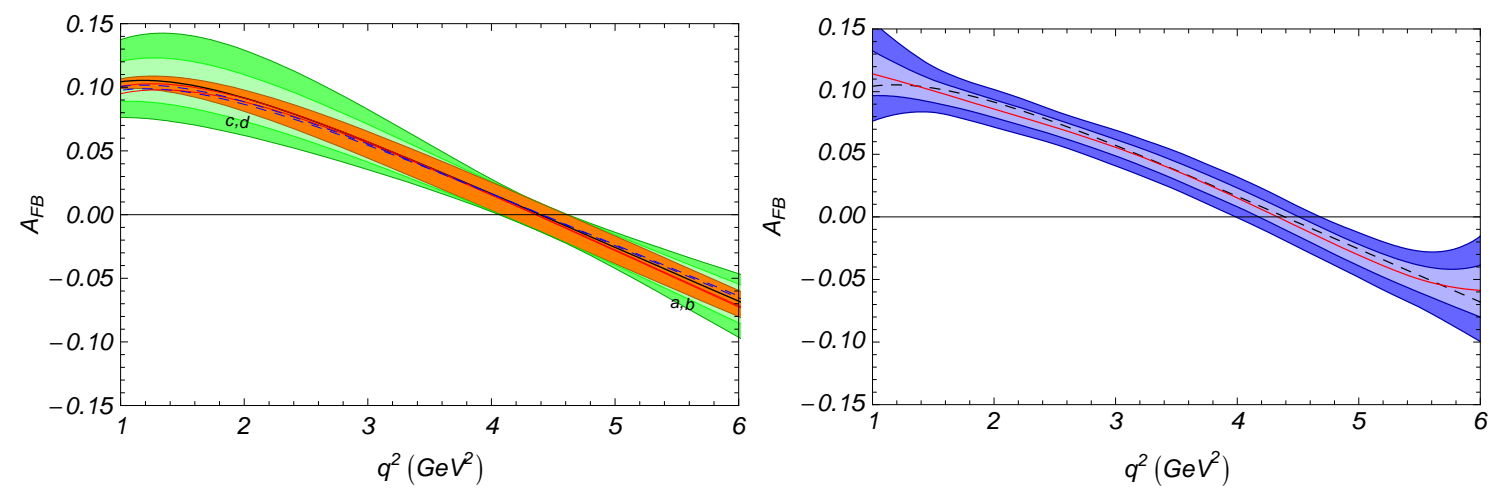

Figure 6: For $A_{F B}$ we compare the theoretical errors (left) with the experimental errors (right). See the caption of Fig. 3 for details.
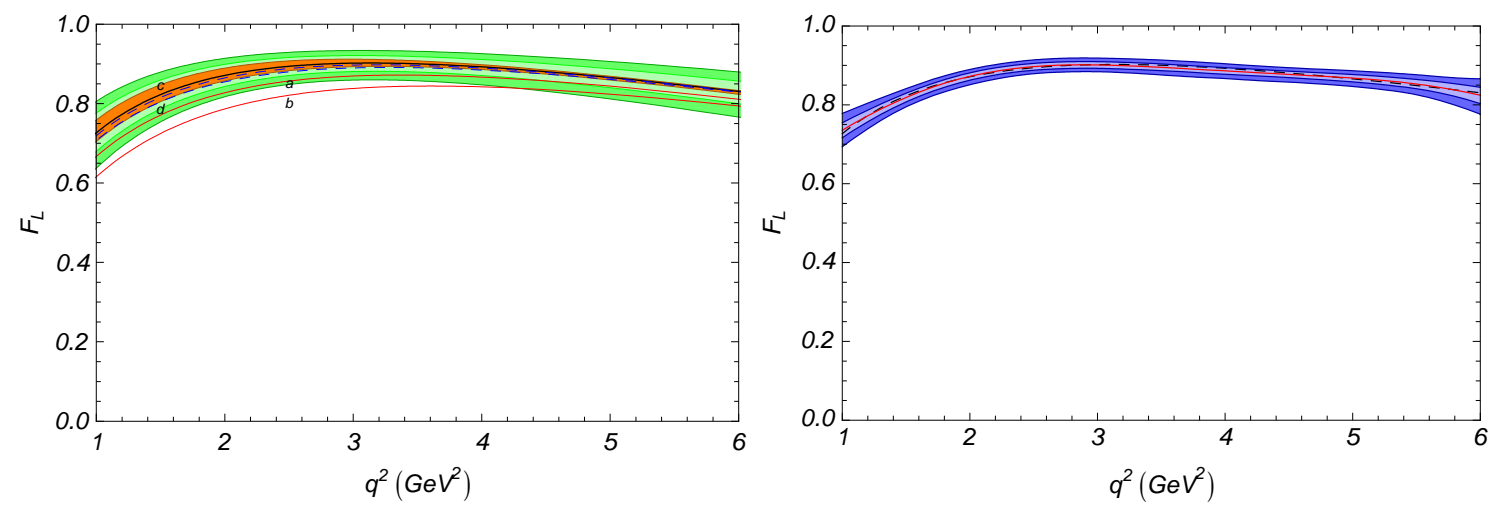

Figure 7: For $F_{L}$ we compare the theoretical errors (left) with the experimental errors (right). See the caption of Fig. 3 for details.

$A_{T}^{(2)}, A_{T}^{(3)}$ and $A_{T}^{(4)}$ versus the old observables like $F_{L}$ concerns the potential discovery of NP, in particular of new right-handed currents. The new observables share the nice feature of $A_{T}^{(2)}$ that there are large deviations from the SM curve from the ones of the four supersymmetric benchmark points. In case of $A_{T}^{(2)}$ this is caused by the balance between the competing contributions of order $1 / q^{2}$ and $1 / q^{4}$ originating from the photon pole in the numerator and denominator of $A_{T}^{(2)}$, providing a strong sensitivity to $\mathcal{C}_{7}^{\prime(\text { eff })}$. This sensitivity is near maximal around the $1 \mathrm{GeV}^{2}$ region precisely inside the theoretically well controlled area. A large deviation from the SM for $A_{T}^{(2)}, A_{T}^{(3)}$ or $A_{T}^{(4)}$ can thus show the presence of right-handed currents in a way that is not possible with $F_{L}$ or $A_{F B}$. In the latter cases the deviations from the SM prediction of the same four representative curves are marginal.

In the experimental plots we find a good agreement between the central values extracted from the fits and the theoretical input. Any deviations seen are small compared to the statistical uncertainties, however the weakness of the polynomial parametrisation, particularly at the extremes of the $q^{2}$ range, can be seen. For much larger data sets this could be addressed by increasing the order of the polynomials used. The experimental 
resolution for $F_{L}$ is very good but with the small deviations from the SM expected this is not helpful in the discovery of new right-handed currents. Comparing the theoretical and experimental figures for the other observables it can be seen that in particular $A_{T}^{(3)}$ show great promise to distinguish between NP models.

To further explore the power of the observables we can imagine that nature corresponds to SUSY scenario (b). We create an ensemble of datasets from the toy Monte Carlo model assuming model (b) as input and compare the results to the SM prediction including the theoretical errors to get a feeling for how significantly different from the SM prediction the results are. The results of this are presented in Figs. 810: It can be seen that $A_{T}^{(2)}, A_{T}^{(3)}$ and $A_{T}^{(4)}$ all show a remarkable separation between the experimental error band and the SM prediction thus providing high sensitivity to NP. For the SUSY scenario (b) chosen here, the deviation for $A_{\mathrm{FB}}$ and $F_{L}$ on the other hand is minor.
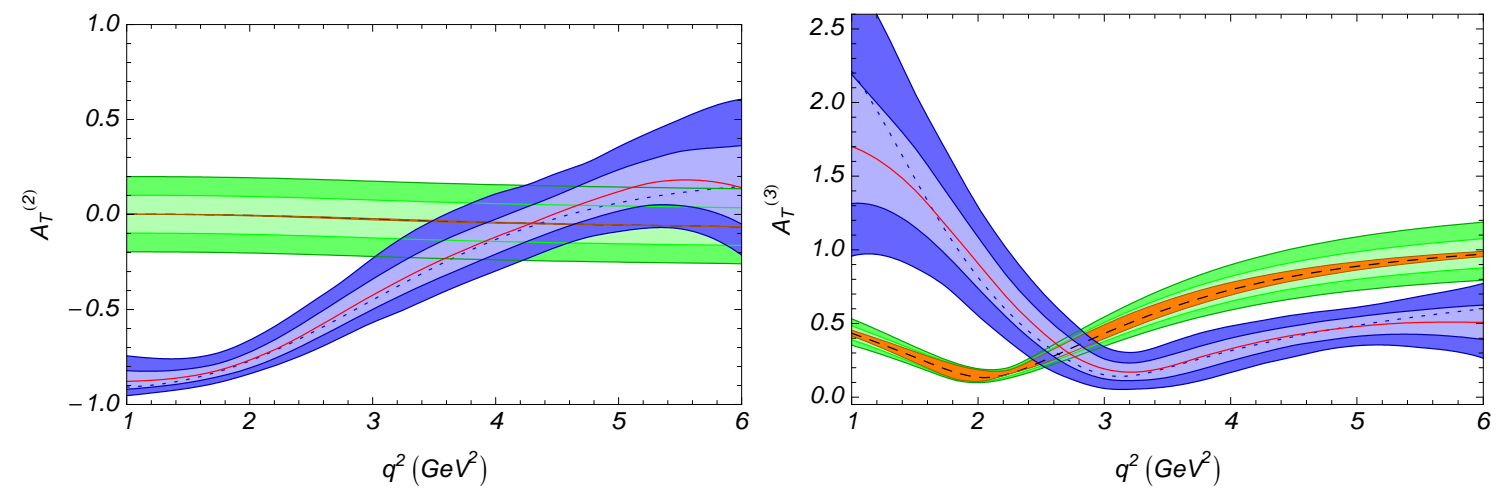

Figure 8: The experimental errors (blue, on top) assuming SUSY scenario (b) with large-gluino mass and positive mass insertion, is compared to the theoretical errors (green, below) assuming the SM. To the left for $A_{T}^{(2)}$ and the right for $A_{T}^{(3)}$.

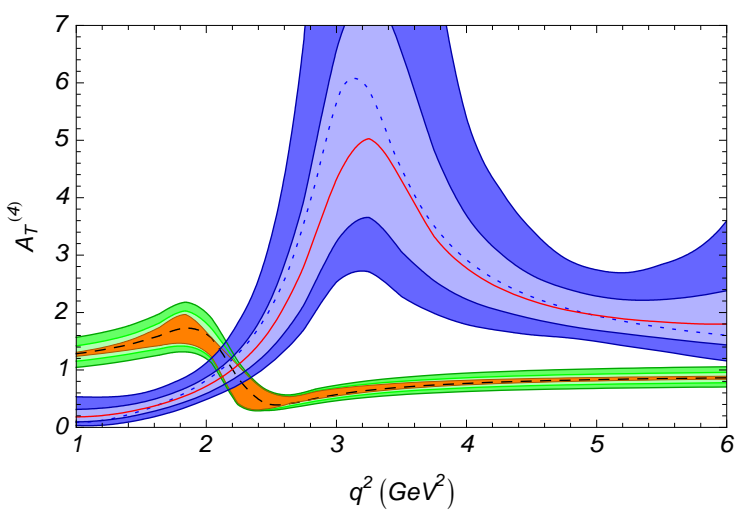

Figure 9: The experimental errors (blue, on top) assuming SUSY scenario (b) with large-gluino mass and positive mass insertion, is compared to the theoretical errors (green, below) assuming the SM. Here the observable $A_{T}^{(4)}$ is considered. 

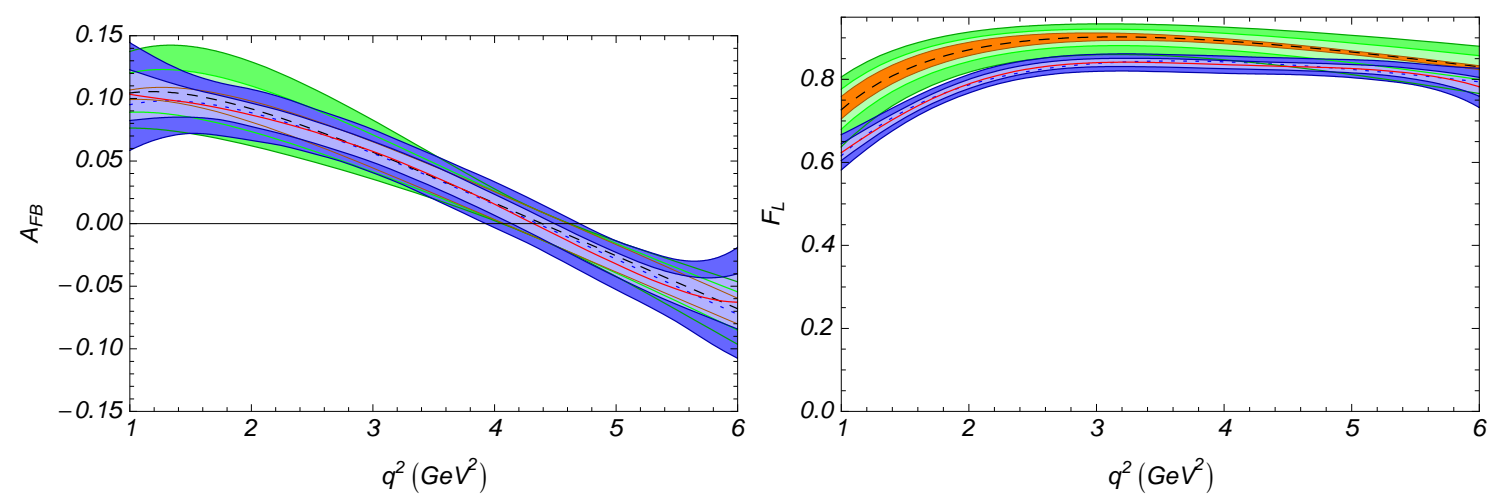

Figure 10: The experimental errors (blue, on top) assuming SUSY scenario (b) with large-gluino mass and positive mass insertion, is compared to the theoretical errors (green, below) assuming the SM. To the left for $A_{\mathrm{FB}}$ and the right for $F_{L}$.

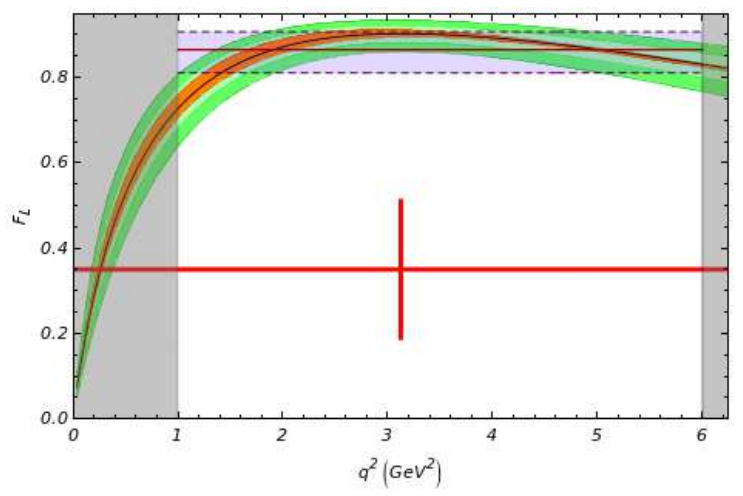

Figure 11: Weighted SM average over the bin $q^{2} \in\left[1 \mathrm{GeV}^{2}, 6 \mathrm{GeV}^{2}\right]$ and recent $B A B A R$ measurement using the extended bin $q^{2} \in\left[4 m_{\mu}^{2}, 6.25 \mathrm{GeV}^{2}\right]$ (shown in grey).

As mentioned in the introduction, the $B$ factories can already access some of the angular observables using the projection-fit method described in Sec. 5. For example, recently the $B A B A R$ collaboration announced the first measurement of the longitudinal polarisation in the low $q^{2}$ region as an average over the bin $q^{2} \in\left[4 m_{\mu}^{2}, 6.25 \mathrm{GeV}^{2}\right]$ [8] (see Fig. 11):

$$
F_{L}\left(q^{2} \in\left[4 m_{\mu}^{2}, 6.25 \mathrm{GeV}^{2}\right]\right)=0.35 \pm 0.16_{\text {stat }} \pm 0.04_{\text {syst }} .
$$

However, as mentioned before, the spectrum below $1 \mathrm{GeV}^{2}$ is theoretically problematic; moreover the rate and also the polarisation $F_{L}$ are changing dramatically around $1 \mathrm{GeV}^{2}$. Therefore, we strongly recommend to use the standard bin from $1 \mathrm{GeV}^{2}$ to $6 \mathrm{GeV}^{2}$. For future comparison we give here the theoretical average, weighted over the rate, using the bin, $q^{2} \in\left[1 \mathrm{GeV}^{2}, 6 \mathrm{GeV}^{2}\right]$, based on our results:

$$
F_{L}\left(q^{2} \in\left[1 \mathrm{GeV}^{2}, 6 \mathrm{GeV}^{2}\right]\right)=0.86 \pm 0.05 .
$$

and refer to Fig. 7 for the future experimental sensitivity of the LHC $b$ experiment. In Fig. 11 we see the theoretical $q^{2}$ distribution of $F_{\mathrm{L}}$ with the rate average overlaid. 

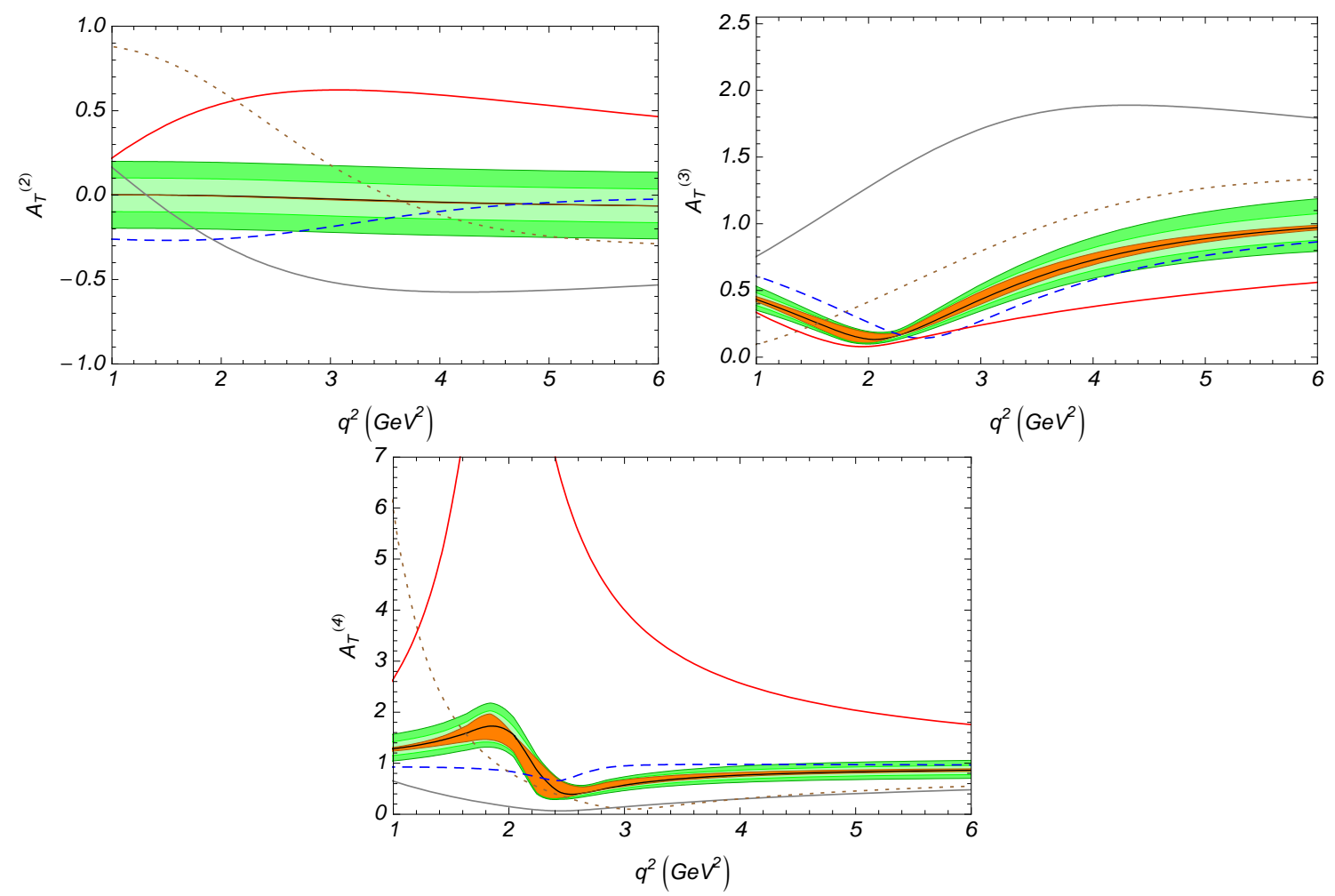

Figure 12: The distribution of $A_{T}^{(2)}, A_{T}^{(3)}$ and $A_{T}^{(4)}$ for four allowed combinations of $\mathcal{C}_{7}^{(\text {eff })}$ and $\mathcal{C}_{7}^{\prime}$ (eff) following the model independent analysis of [21]. The bands correspond to the SM and the theoretical uncertainty as described in Fig 3. The solid heavy (red) line corresponds to $\left(\mathcal{C}_{7}^{\text {(eff) }}, \mathcal{C}_{7}^{\prime}{ }^{(\text {eff })}\right)=(0.04,0.31)$, the solid light (grey) line $(-0.03,-0.32)$, the dashed (blue) line $(-0.35,0.05)$, and the dotted (brown) line $(-0.24,-0.19)$. Combining measurements in all three asymmetries will provide clear distinction between the different allowed regions.

Rather than using the benchmark supersymmetry points for the illustration of the power of the observables, one can also look at it from a model independent point of view. For this we have taken four illustrative points from Fig. 2 in [21] which are all allowed given the constraints from present measurements of $b \rightarrow s$ transitions. In Fig. 12 the effect can be seen on $A_{T}^{(2)}, A_{T}^{(3)}$ and $A_{T}^{(4)}$. It is clear that the combination of all observables will act as a way to reduce the allowed regions for a model independent analysis.

Finally we might ask what happens if we consider the situation with $100 \mathrm{fb}^{-1}$ of experimental data corresponding to the full dataset from an upgrade to LHC $b$. We assume the same performance of the experiment so simply scale the statistics by a factor 10 compared to the $10 \mathrm{fb}^{-1}$ study. The experimental errors are shown in Fig. 13 and are in general just a factor $\sqrt{10}$ smaller as expected. Comparing to Figs. 1 and 5 it can be seen that the $\Lambda / m_{b}$ uncertainties will dominate unless progress is made on the theoretical side.

\section{Summary}

We have constructed two new observables $A_{T}^{(3)}$ and $A_{T}^{(4)}$ out of the $K^{*}$ spin amplitudes of 

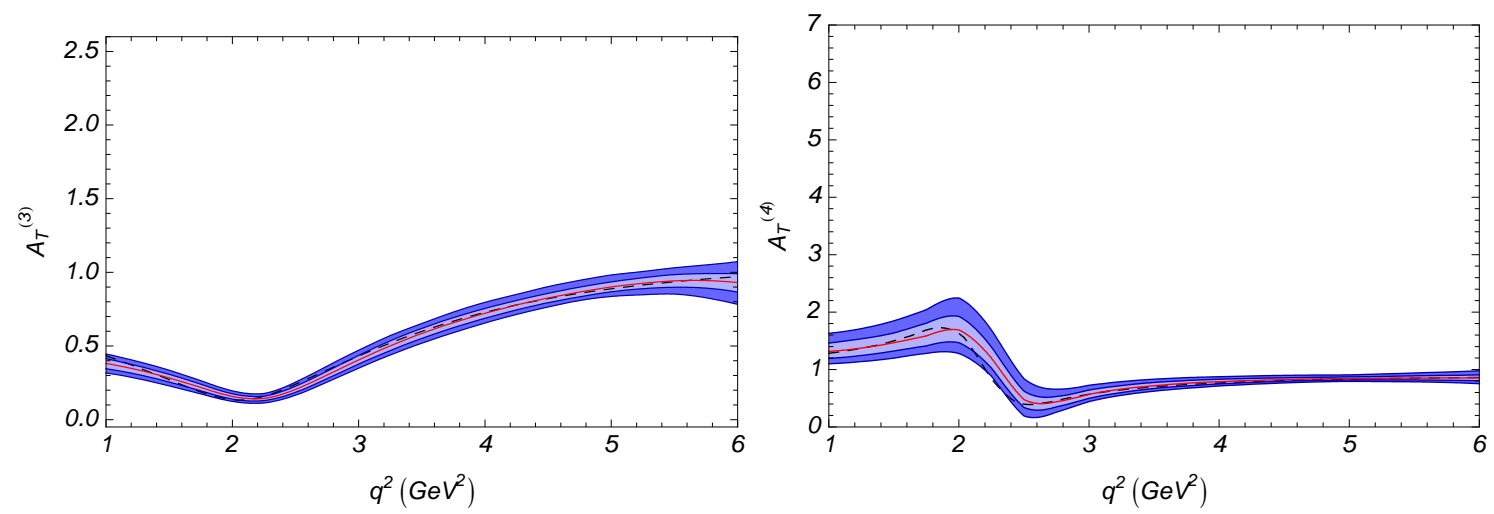

Figure 13: The experimental errors in $A_{T}^{(3)}$ (right) and $A_{T}^{(4)}$ left assuming the SM for statistics equivalent to $100 \mathrm{fb}^{-1}$ at the end of an upgrade to LHC $b$.

the $\bar{B}_{d} \rightarrow \bar{K}^{* 0} \mu^{+} \mu^{-}$decay, that fulfil the criteria of being theoretically clean and can be experimentally extracted from the angular distribution of this decay with good precision. We have shown how to design the new observables for a specific kind of NP operator within the model independent analysis using the effective field theory approach.

We have presented a complete calculation of all observables in QCD factorization and have made the impact of unknown $\Lambda / m_{b}$ corrections to the various observables explicit. Subsequently, we demonstrated the high sensitivity of $A_{T}^{(2)}, A_{T}^{(3)}$ and $A_{T}^{(4)}$ to right handed currents. Clearly theoretical progress on the $\Lambda / m_{b}$ corrections would enhance that sensitivity significantly and would be desirable in view of an upgrade of the LHC $b$ experiment.

The new observables $A_{T}^{(3)}$ and $A_{T}^{(4)}$ exhibit the important property of presenting a direct sensitivity to the longitudinal spin amplitude, while reducing at maximum the sensitivity to the poorly known longitudinal soft form factors within the whole low dilepton mass spectrum. Previously defined $F_{L}$ or $A_{\mathrm{FB}}$ does not exhibit this behaviour. This same idea was behind the construction of $A_{T}^{(2)}$ using the transverse amplitudes.

The combination of the three observables offer a full view of the sensitivity to NP of the three spin amplitudes with a good control of hadronic uncertainties.

Using a toy Monte Carlo approach we have estimated the statistical uncertainty of all observables for statistics corresponding to LHC $b$ and also for Super-LHC $b$. The model performs a fit to the full angular and $q^{2}$ distribution. $A_{T}^{(3)}$ and $A_{T}^{(4)}$ require a full angular fit and for $A_{T}^{(2)}$ we have demonstrated that the resolution improves by more than a factor 2 compared to extracting $A_{T}^{(2)}$ from angular projection. The experimental errors are such that measuring these new observables will be a powerful way to detect the presence of right handed currents. For the well known measurement of the zero point of the forwardbackward asymmetry we see an improvement of $30 \%$ in the resolution from a full angular fit compared to fitting the angular projections.

Finally we have shown that the previously discussed angular distribution $A_{T}^{(1)}$ cannot be measured at either LHC $b$ or at a Super- $B$ factory. 


\section{Acknowledgments}

We thank Martin Beneke for detailed discussions on the forward-backward asymmetry. JM acknowledges financial support from FPA2005-02211, 2005-SGR-00994 and the RyC programme, MR from the Universitat Autonoma de Barcelona, and UE and WR from the Science and Technology Facilities Council (STFC). TH acknowledges support of the European network Heptools. 


\section{A. Kinematics}

Assuming the $K^{*}$ to be on the mass shell, the decay $\bar{B}^{0} \rightarrow \bar{K}^{* 0}\left(\rightarrow K^{-} \pi^{+}\right) \ell^{+} \ell^{-}$is completely described by four independent kinematic variables; namely, the lepton-pair invariant mass, $q^{2}$, and the three angles $\theta_{l}, \theta_{K^{*}}, \phi$ as illustrated in Fig. 14. The sign of the angles for the $\bar{B}_{d}$ decay shows great variation in the literature. Therefore we present here the most explicit definition of our conventions. Here $\mathbf{p}$ denote three momentum vectors in the $\bar{B}_{d}$

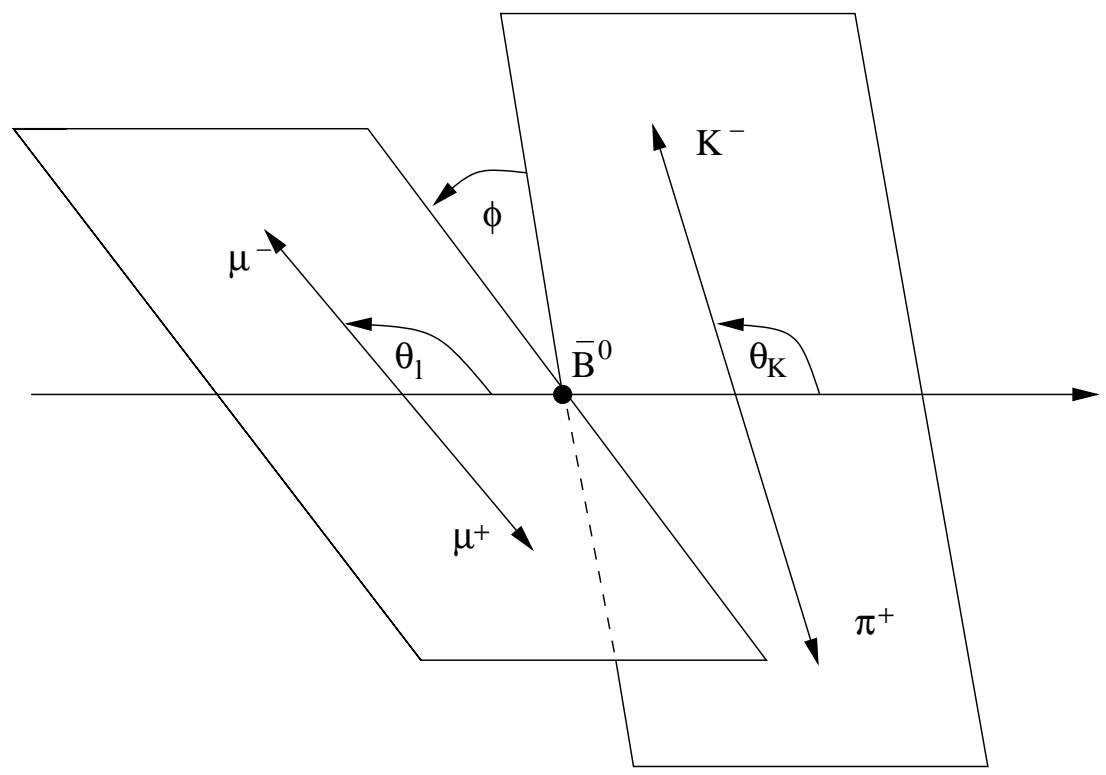

Figure 14: Definition of kinematic variables in the decay $\bar{B}_{d} \rightarrow \bar{K}^{* 0} \mu^{+} \mu^{-}$: The $z$-axis is the direction in which the $B$ meson flies in the rest frame of the $\mu^{+} \mu^{-} . \theta_{l}$ is the angle between the $\mu^{-}$ and the $z$-axis in the $\mu^{+} \mu^{-}$rest frame, $\theta_{K}$ is the angle between the $K^{-}$and the $z$-axis in the $\bar{K}^{*}$ rest frame, and $\phi$ is the angle between the normals to the $\mu^{+} \mu^{-}$and $K \pi$ decay planes in the $B$ rest frame.

rest frame, $\mathbf{q}$ the same in the di-muon rest frame, and $\mathbf{r}$ in the $\bar{K}^{* 0}$ rest frame, the $z$-axis is defined as as the direction of the $\bar{K}^{* 0}$ in the $\bar{B}_{d}$ rest frame. Three unit vectors are given in the following way: the first one is in the direction of the $z$-axis where the $\theta$ angles are measured with respect to, and the other two are perpendicular to the di-muon and $\bar{K}^{* 0}$ decay planes.

$$
\mathbf{e}_{z}=\frac{\mathbf{p}_{K^{-}}+\mathbf{p}_{\pi^{+}}}{\left|\mathbf{p}_{K^{-}}+\mathbf{p}_{\pi^{+}}\right|}, \quad \mathbf{e}_{l}=\frac{\mathbf{p}_{\mu^{-}} \times \mathbf{p}_{\mu^{+}}}{\left|\mathbf{p}_{\mu^{-}} \times \mathbf{p}_{\mu^{+}}\right|}, \quad \mathbf{e}_{K}=\frac{\mathbf{p}_{K^{-}} \times \mathbf{p}_{\pi^{+}}}{\left|\mathbf{p}_{K^{-}} \times \mathbf{p}_{\pi^{+}}\right|}
$$

It follows for the $\bar{B}_{d}$

$$
\cos \theta_{l}=\frac{\mathbf{q}_{\mu^{-}} \cdot \mathbf{e}_{z}}{\left|\mathbf{q}_{\mu^{-}}\right|}, \quad \cos \theta_{K}=\frac{\mathbf{r}_{K^{-}} \cdot \mathbf{e}_{z}}{\left|\mathbf{r}_{K^{-}}\right|}
$$

and

$$
\sin \phi=\left(\mathbf{e}_{l} \times \mathbf{e}_{K}\right) \cdot \mathbf{e}_{z}, \quad \cos \phi=\mathbf{e}_{K} \cdot \mathbf{e}_{l} .
$$

The angles are defined in the intervals

$$
-1 \leqslant \cos \theta_{l} \leqslant 1, \quad-1 \leqslant \cos \theta_{K} \leqslant 1, \quad-\pi \leqslant \phi<\pi,
$$


where in particular it should be noted that the $\phi$ angle is signed.

In words, for the $\bar{B}_{d}$ the angle $\theta_{l}$ is measured as the angle between the $\mu^{-}$and the $z$-axis in the dimuon rest frame. As the $\bar{B}_{d}$ flies in the direction of the $z$-axis in the dimuon rest frame this is equivalent to measuring $\theta_{l}$ as the angle between the muon and the $\bar{B}_{d}$ in the di-muon rest frame. The angle $\theta_{K}$ is measured as the angle between the kaon and the $z$-axis measured in the $\bar{K}^{* 0}$ rest frame. Finally $\phi$ is the angle between the normals to the planes defined by the $K \pi$ system and the $\mu^{+} \mu^{-}$system in the rest frame of the $\bar{B}_{d}$ meson.

\section{B. Theoretical framework}

The coefficient functions $I_{i}$ in the differential decay rate are given in terms of the $K^{*}$ spin amplitudes (see Eq. (2.3)) discussed in Sec. 3. The theoretical expressions of those spin amplitudes can be derived using the following standard steps:

- The effective Hamiltonian describing the quark transition $b \rightarrow s \ell^{+} \ell^{-}$is given by

$$
\mathcal{H}_{\mathrm{eff}}=-\frac{4 G_{F}}{\sqrt{2}} V_{t b} V_{t s}^{*} \sum_{i=1}^{10}\left[C_{i}(\mu) \mathcal{O}_{i}(\mu)+C_{i}^{\prime}(\mu) \mathcal{O}_{i}^{\prime}(\mu)\right],
$$

where in addition to the SM operators we have also added the chirally flipped partners. In what follows, the same conventions are used as in [19]. In the NP analysis, we will focus on the the chirally flipped $\mathcal{O}_{7}^{\prime}$ operator in addition to the most important SM operators $\mathcal{O}_{7}, \mathcal{O}_{9}$, and $\mathcal{O}_{10}$ :

$$
\begin{array}{ll}
\mathcal{O}_{7}=\frac{e}{16 \pi^{2}} m_{b}\left(\bar{s} \sigma_{\mu \nu} P_{R} b\right) F^{\mu \nu}, & \mathcal{O}_{7}^{\prime}=\frac{e}{16 \pi^{2}} m_{b}\left(\bar{s} \sigma_{\mu \nu} P_{L} b\right) F^{\mu \nu}, \\
\mathcal{O}_{9}=\frac{e^{2}}{16 \pi^{2}}\left(\bar{s} \gamma_{\mu} P_{L} b\right)\left(\bar{\ell} \gamma^{\mu} \ell\right), & \mathcal{O}_{10}=\frac{e^{2}}{16 \pi^{2}}\left(\bar{s} \gamma_{\mu} P_{L} b\right)\left(\bar{\ell} \gamma^{\mu} \gamma_{5} \ell\right),
\end{array}
$$

where $P_{L, R}=\left(1 \mp \gamma_{5}\right) / 2$ and $m_{b} \equiv m_{b}(\mu)$ is the running mass in the $\overline{\mathrm{MS}}$ scheme.

- The hadronic part of the matrix element describing the $B \rightarrow K \pi$ transition can be parameterised in terms of $B \rightarrow K^{*}$ form factors by means of a narrow-width approximation (see for example [22]). The relevant form factors are defined as:

$$
\begin{aligned}
&\left\langle K^{*}\left(p_{K^{*}}\right)\left|\bar{s} \gamma_{\mu} P_{L, R} b\right| B(p)\right\rangle=i \epsilon_{\mu \nu \alpha \beta} \epsilon^{\nu *} p^{\alpha} q^{\beta} \frac{V\left(q^{2}\right)}{m_{B}+m_{K^{*}}} \mp \\
& \mp \frac{1}{2}\left\{\epsilon_{\mu}^{*}\left(m_{B}+m_{K^{*}}\right) A_{1}\left(q^{2}\right)-\left(\epsilon^{*} \cdot q\right)(2 p-q)_{\mu} \frac{A_{2}\left(q^{2}\right)}{m_{B}+m_{K^{*}}}-\right. \\
&\left.\quad-\frac{2 m_{K^{*}}}{q^{2}}\left(\epsilon^{*} \cdot q\right)\left[A_{3}\left(q^{2}\right)-A_{0}\left(q^{2}\right)\right] q_{\mu}\right\}
\end{aligned}
$$

where

$$
A_{3}\left(q^{2}\right)=\frac{m_{B}+m_{K^{*}}}{2 m_{K^{*}}} A_{1}\left(q^{2}\right)-\frac{m_{B}-m_{K^{*}}}{2 m_{K^{*}}} A_{2}\left(q^{2}\right),
$$


and

$$
\begin{array}{r}
\left\langle K^{*}\left(p_{K^{*}}\right)\left|\bar{s} i \sigma_{\mu \nu} q^{\nu} P_{R, L} b\right| B(p)\right\rangle=-i \epsilon_{\mu \nu \alpha \beta} \epsilon^{\nu *} p^{\alpha} q^{\beta} T_{1}\left(q^{2}\right) \pm \\
\pm \frac{1}{2}\left\{\left[\epsilon_{\mu}^{*}\left(m_{B}^{2}-m_{K^{*}}^{2}\right)-\left(\epsilon^{*} \cdot q\right)(2 p-q)_{\mu}\right] T_{2}\left(q^{2}\right)+\right. \\
\left.+\left(\epsilon^{*} \cdot q\right)\left[q_{\mu}-\frac{q^{2}}{m_{B}^{2}-m_{K^{*}}^{2}}(2 p-q)_{\mu}\right] T_{3}\left(q^{2}\right)\right\} .
\end{array}
$$

In the above, $q=p_{l^{+}}+p_{l^{-}}$and $\epsilon^{\mu}$ is the $K^{*}$ polarisation vector.

- In the heavy-quark and large-energy limit the seven a priori independent $B \rightarrow K^{*}$ form factors in Eqs. (B.4) and (B.6) reduce to two universal form factors $\xi_{\perp}$ and $\xi_{\|}$ in the leading order [27, 28]: ${ }^{4}$

$$
\begin{gathered}
A_{1}\left(q^{2}\right)=\frac{2 E_{K^{*}}}{m_{B}+m_{K^{*}}} \xi_{\perp}\left(E_{K^{*}}\right), \\
A_{2}\left(q^{2}\right)=\frac{m_{B}}{m_{B}-m_{K^{*}}}\left[\xi_{\perp}\left(E_{K^{*}}\right)-\xi_{\|}\left(E_{K^{*}}\right)\right] \\
A_{0}\left(q^{2}\right)=\frac{E_{K^{*}}}{m_{K^{*}}} \xi_{\|}\left(E_{K^{*}}\right), \\
V\left(q^{2}\right)=\frac{m_{B}+m_{K^{*}}}{m_{B}} \xi_{\perp}\left(E_{K^{*}}\right) \\
T_{1}\left(q^{2}\right)=\xi_{\perp}\left(E_{K^{*}}\right) \\
T_{2}\left(q^{2}\right)=\frac{2 E_{K^{*}}}{m_{B}} \xi_{\perp}\left(E_{K^{*}}\right) \\
T_{3}\left(q^{2}\right)=\xi_{\perp}\left(E_{K^{*}}\right)-\xi_{\|}\left(E_{K^{*}}\right) .
\end{gathered}
$$

Here, $E_{K^{*}}$ is the energy of the final vector meson in the $B$ rest frame,

$$
E_{K^{*}} \simeq \frac{m_{B}}{2}\left(1-\frac{q^{2}}{m_{B}^{2}}\right)
$$

These relations, valid in the low- $q^{2}$ region, allow to simplify the spin amplitudes to obtain Eqs. (3.7 3.9) which are crucial for the construction of our new observables. They are violated by symmetry breaking corrections of order $\alpha_{s}$ and $1 / m_{b}$.

\section{NLO corrections to the spin amplitudes}

The NLO corrections to the form factors at order $\alpha_{s}$ are given in Ref. [17]. In the presence of right-handed currents $\left(\mathcal{C}_{7}^{\prime(\text { eff })} \neq 0\right)$, the spin amplitudes read [19, 20]

$$
A_{\perp L, R}=N \sqrt{2} \lambda^{1 / 2}\left[\left(\mathcal{C}_{9} \mp \mathcal{C}_{10}\right) \frac{V\left(q^{2}\right)}{m_{B}+m_{K^{*}}}+\frac{2 m_{b}}{q^{2}} \mathcal{T}_{\perp \mathrm{NLO}}^{+}\left(q^{2}\right)\right]
$$

${ }^{4}$ Following Ref. [28], the longitudinal form factor $\xi_{\|}$is related to that of Ref. [27] by $\xi_{\|}=\left(m_{K^{*}} / E_{K^{*}}\right) \zeta_{\|}$. 


$$
\begin{aligned}
A_{\| L, R}=- & N \sqrt{2}\left(m_{B}^{2}-m_{K^{*}}^{2}\right)\left[\left(\mathcal{C}_{9} \mp \mathcal{C}_{10}\right) \frac{A_{1}\left(q^{2}\right)}{m_{B}-m_{K^{*}}}+\frac{4 m_{b} E_{K^{*}}}{m_{B} \cdot s} \mathcal{T}_{\perp \mathrm{NLO}}^{-}\left(q^{2}\right)\right] \\
A_{0 L, R}=- & \frac{N}{2 m_{K^{*}} q} \times \\
\times & {\left[\left(\mathcal{C}_{9} \mp \mathcal{C}_{10}\right)\left\{\left(m_{B}^{2}-m_{K^{*}}^{2}-q^{2}\right)\left(m_{B}+m_{K^{*}}\right) A_{1}\left(q^{2}\right)-\lambda \frac{A_{2}\left(q^{2}\right)}{m_{B}+m_{K^{*}}}\right\}\right.} \\
& 2 m_{b}\left\{\left(m_{B}^{2}+3 m_{K^{*}}^{2}-q^{2}\right) \frac{2 E_{K^{*}}}{m_{B}} \mathcal{T}_{\perp \mathrm{NLO}}^{-}\left(q^{2}\right)\right. \\
& \left.\left.-\frac{\lambda}{m_{B}^{2}-m_{K^{*}}^{2}}\left(\mathcal{T}_{\perp \mathrm{NLO}}^{-}\left(q^{2}\right)+\mathcal{T}_{\| \mathrm{NLO}}^{-}\left(q^{2}\right)\right)\right\}\right]
\end{aligned}
$$

where $\lambda$ is defined in Eq. 3.5 and the form factor relations for $V\left(q^{2}\right), A_{0}\left(q^{2}\right)$ and $A_{1}\left(q^{2}\right)$ are as in Eq. 3.7. $A_{2}\left(q^{2}\right)$ is given by

$$
A_{2}\left(q^{2}\right)=\frac{m_{B}}{m_{B}-m_{K^{*}}}\left[\xi_{\perp}\left(q^{2}\right)-\xi_{\|}\left(q^{2}\right)(1-C)\right],
$$

with $C$ the $\mathcal{O}\left(\alpha_{s}\right)$ correction to the form factor $A_{2}$ computed in [28, 17, 35]:

$$
C=\frac{\alpha_{s}}{3 \pi}\left[(2-2 L)+8 \frac{m_{K^{*}}}{E_{K^{*}}} \frac{m_{B}\left(m_{B}-2 E_{K^{*}}\right)}{4 E_{K^{*}}^{2}} \kappa_{\|}\left(q^{2}\right) \lambda_{B,+}^{-1} \int_{0}^{1} d u \frac{\Phi_{K^{*}, \|}(u)}{1-u}\right]
$$

with $\Phi_{K^{*}, \|}(u)$ being the longitudinal light-cone distribution amplitude of the vector meson $\bar{K}^{* 0}$. Moreover, we have

$$
\begin{aligned}
\mathcal{T}_{\perp \mathrm{NLO}}^{ \pm}= & \xi_{\perp}\left(q^{2}\right)\left\{C_{\perp}^{(0, \pm)}+\frac{\alpha_{s}}{3 \pi}\left[C_{\perp}^{(1, \pm)}+\right.\right. \\
& \left.\left.+\kappa_{\perp}\left(q^{2}\right) \lambda_{B,+}^{-1} \int_{0}^{1} d u \Phi_{K^{*}, \perp}(u)\left[T_{\perp,+}^{(\mathrm{f} \pm)}(u)+T_{\perp,+}^{(\mathrm{nf})}(u)\right]\right]\right\}
\end{aligned}
$$

and

$$
\begin{aligned}
& \mathcal{T}_{\| \mathrm{NLO}}^{ \pm}=\xi_{\|}\left(q^{2}\right)\left\{C_{\|}^{(0, \pm)}+\kappa_{\|}\left(q^{2}\right) \frac{m_{K}^{*}}{E_{K^{*}}} \lambda_{B,-}^{-1}\left(q^{2}\right) \int_{0}^{1} d u \Phi_{K^{*}, \|}(u) \hat{T}_{\|,-}^{(0)}(u)+\right. \\
& +\frac{\alpha_{s}}{3 \pi}\left[C_{\|}^{(1, \pm)}+\kappa_{\|}\left(q^{2}\right) \frac{m_{K}^{*}}{E_{K^{*}}}\left(\lambda_{B,+}^{-1} \int_{0}^{1} d u \Phi_{K^{*}, \|}(u)\left[T_{\|,+}^{(\mathrm{f} \pm)}(u)+T_{\|,+}^{(\mathrm{nf})}(u)\right]+\right.\right. \\
& \left.\left.\left.+\lambda_{B,-}^{-1}\left(q^{2}\right) \int_{0}^{1} d u \Phi_{K^{*}, \|}(u) \hat{T}_{\|,-}^{(\mathrm{nf})}(u)\right)\right]\right\},
\end{aligned}
$$

where $\kappa_{z} \equiv \pi^{2} f_{B} f_{K^{*}, z}(\mu) /\left(N_{c} m_{B} \xi_{z}\left(q^{2}\right)\right.$ ) (with $\left.z=\perp, \|\right) . \quad \lambda_{B,+}^{-1}$ and $\lambda_{B,-}^{-1}\left(q^{2}\right)$ are the two $\bar{B}_{d}$ meson light-cone distribution amplitude moments defined in [17]; they are given by

$$
\begin{aligned}
\lambda_{B,+}^{-1} & =\int_{0}^{\infty} d \omega \frac{\Phi_{B,+}(\omega)}{\omega}, \\
\lambda_{B,-}^{-1}\left(q^{2}\right) & =\int_{0}^{\infty} d \omega \frac{\Phi_{B,-}(\omega)}{\omega-q^{2} / m_{B}-i \epsilon} .
\end{aligned}
$$


In all cases the symbol + stands for the substitution of $\mathcal{C}_{7}^{(\text {eff })} \rightarrow \mathcal{C}_{7}^{(\text {eff })}+\mathcal{C}_{7}^{\text {(eff) }}$ and - for $\mathcal{C}_{7}^{(\text {eff })} \rightarrow \mathcal{C}_{7}^{(\text {eff })}-\mathcal{C}_{7}^{\prime}($ eff $)$, wherever $\mathcal{C}_{7}^{(\text {eff })}$ appears. For instance, in the definition of

$$
C_{z}^{(1, \pm)}=C_{z}^{(\mathrm{f} \pm)}+C_{z}^{(\mathrm{nf})}
$$

with $z=\perp, \|$, the factorizable correction reads [28, 17]

$$
\begin{aligned}
& C_{\perp}^{(\mathrm{f} \pm)}=\left(C_{7}^{\mathrm{eff}} \pm C_{7}^{\mathrm{eff} \prime}\right)\left(4 \ln \frac{m_{b}^{2}}{\mu^{2}}-4-L\right), \\
& C_{\|}^{(\mathrm{f} \pm)}=-\left(C_{7}^{\mathrm{eff}} \pm C_{7}^{\mathrm{eff} \prime}\right)\left(4 \ln \frac{m_{b}^{2}}{\mu^{2}}-6+4 L\right)+\frac{m_{B}}{2 m_{b}} Y\left(q^{2}\right)(2-2 L)
\end{aligned}
$$

with

$$
L \equiv-\frac{m_{b}^{2}-q^{2}}{q^{2}} \ln \left(1-\frac{q^{2}}{m_{b}^{2}}\right)
$$

while the non-factorizable contribution $C_{z}^{(\mathrm{nf})}$ is common to both. In the definition of the hard scattering functions with $T_{z, \pm}^{(1 \pm)}=T_{z, \pm}^{(\mathrm{f} \pm)}+T_{z, \pm}^{(\mathrm{nf})}$, the factorizable correction reads [28, 17]:

$$
\begin{aligned}
& T_{\perp,+}^{(\mathrm{f} \pm)}(u, \omega)=\left(C_{7}^{\mathrm{eff}} \pm C_{7}^{\mathrm{eff} \prime}\right) \frac{2 m_{B}}{(1-u) E_{K^{*}}}, \\
& T_{\|,+}^{(\mathrm{f} \pm)}(u, \omega)=\left[\left(C_{7}^{\mathrm{eff}} \pm C_{7}^{\mathrm{eff} \prime}\right)+\frac{q^{2}}{2 m_{b} m_{B}} Y\left(q^{2}\right)\right] \frac{2 m_{B}^{2}}{(1-u) E_{K^{*}}^{2}} \\
& T_{\perp,-}^{(\mathrm{f})}(u, \omega)=T_{\|,-}^{(\mathrm{f})}(u, \omega)=0 .
\end{aligned}
$$

Again the non-factorizable part is common to both cases, because it does not receive contributions from $\mathcal{O}_{7}$. For the definition of the function $Y\left(q^{2}\right)$ and for the non-factorizable contributions we refer the reader to [28, 17].

\section{References}

[1] M. Artuso et al., $B, D$ and $K$ decays, arXiv:0801.1833,

[2] T. Hurth, Present status of inclusive rare B decays, Rev. Mod. Phys. $\mathbf{7 5}$ (2003) 1159 hep-ph/0212304.

[3] T. Hurth, Status of SM calculations of $b \rightarrow s$ transitions, Int. J. Mod. Phys. A 22 (2007) 1781 hep-ph/0703226.

[4] A. Ishikawa et al. [Belle Collaboration], Observation of the electroweak penguin decay $B \rightarrow K^{*} \ell^{+} \ell^{-}$, Phys. Rev. Lett. 91 (2003) 261601 hep-ex/0308044.

[5] B. Aubert et al. [BABAR Collaboration], Evidence for the rare decay $B \rightarrow K^{*} \ell^{+} \ell^{-}$and measurement of the $B \rightarrow K \ell^{+} \ell^{-}$branching fraction, Phys. Rev. Lett. 91 (2003) 221802 hep-ex/0308042.

[6] A. Ishikawa et al., Measurement of forward-backward asymmetry and Wilson coefficients in $B \rightarrow K^{*} \ell^{+} \ell^{-}$, Phys. Rev. Lett. 96 (2006) 251801 hep-ex/0603018. 
[7] B. Aubert et al. [BABAR Collaboration], Measurements of branching fractions, rate asymmetries, and angular distributions in the rare decays $B \rightarrow K \ell^{+} \ell^{-}$and $B \rightarrow K^{*} \ell^{+} \ell^{-}$, Phys. Rev. D 73 (2006) 092001 hep-ex/0604007.

[8] B. Aubert et al. [BABAR Collaboration], Angular Distributions in the Decays $B \rightarrow K^{*} \ell^{+} \ell^{-}$, arXiv:0804.4412.

[9] I. Adachi et al. [The Belle Collaboration], Measurement of the Differential Branching Fraction and Forward-Backward Asymmetry for $B \rightarrow K^{*} \ell^{+} \ell^{-}$, arXiv:0810.0335.

[10] J. Dickens, V. Gibson, C. Lazzeroni and M. Patel, Selection of the decay $B_{d} \rightarrow K^{*} \mu^{+} \mu^{-}$at LHCb , CERN-LHCB-2007-038.

[11] J. Dickens, V. Gibson, C. Lazzeroni and M. Patel, A study of the sensitivity to the forward-backward asymmetry in $B_{d} \rightarrow K^{*} \mu+\mu$ - decays at LHCb, CERN-LHCB-2007-039.

[12] U. Egede, Angular correlations in the $\bar{B}_{d} \rightarrow \bar{K}^{* 0} \mu^{+} \mu^{-}$decay, CERN-LHCB-2007-057.

[13] T. Browder, M. Ciuchini, T. Gershon, M. Hazumi, T. Hurth, Y. Okada and A. Stocchi, On the Physics Case of a Super Flavour Factory, J. High Energy Phys. 02 (2008) 110 arXiv:0710.3799.

[14] M. Bona et al., SuperB: A High-Luminosity Asymmetric $e^{+} e^{-}$Super Flavor Factory. Conceptual Design Report, arXiv:0709.0451.

[15] J. L. . Hewett et al., The discovery potential of a Super B Factory. Proceedings, SLAC Workshops, Stanford, USA, 2003, hep-ph/0503261.

[16] A. G. Akeroyd et al. [SuperKEKB Physics Working Group], Physics at super B factory, hep-ex/0406071.

[17] M. Beneke, T. Feldmann and D. Seidel, Systematic approach to exclusive $B \rightarrow V \ell^{+} \ell^{-}, V \gamma$ decays, Nucl. Phys. B 612 (2001) 25 hep-ph/0106067.

[18] C. H. Chen and C. Q. Geng, Analysis of $B \rightarrow K^{*} \ell^{+} \ell^{-}$decays at large recoil, Nucl. Phys. B 636 (2002) 338 hep-ph/0203003.

[19] F. Kruger and J. Matias, Probing new physics via the transverse amplitudes of $B^{0} \rightarrow K^{* 0}\left(\rightarrow K^{-} \pi^{+}\right) \ell^{+} \ell^{-}$at large recoil, Phys. Rev. D 71 (2005) 094009 hep-ph/0502060; J. Matias, The angular distribution of $B^{0} \rightarrow K^{* 0}\left(\rightarrow K^{-} \pi^{+}\right) \ell^{+} \ell^{-}$at large recoil in and beyond the SM, PoS HEP2005 (2006) 281 hep-ph/0511274.

[20] E. Lunghi and J. Matias, Huge right-handed current effects in $B \rightarrow K^{*}(K \pi) \ell^{+} \ell^{-}$in supersymmetry, J. High Energy Phys. 04 (2007) 058 hep-ph/0612166.

[21] C. Bobeth, G. Hiller and G. Piranishvili, $C P$ Asymmetries in $\bar{B} \rightarrow \bar{K}^{*}(\rightarrow \bar{K} \pi) \ell^{+} \ell^{-}$and untagged $\bar{B}_{s}, B_{s} \rightarrow \phi\left(\rightarrow K^{+} K^{-}\right) \ell^{+} \ell^{-}$decays at $N L O$, arXiv:0805.2525.

[22] F. Krüger, L. M. Sehgal, N. Sinha, and R. Sinha, Angular distribution and CP asymmetries in the decays $\bar{B} \rightarrow K^{-} \pi^{+} e^{-} e^{+}$and $\bar{B} \rightarrow \pi^{-} \pi^{+} e^{-} e^{+}$, Phys. Rev. D 61 (2000) 114028 hep-ph/9907386; ; Phys. Rev. D 63 (2001) 019901(E).

[23] D. Melikhov, N. Nikitin, and S. Simula, Probing right-handed currents in $B \rightarrow K^{*} \ell^{+} \ell^{-}$ transitions, Phys. Lett. B 442 (1998) 381 hep-ph/9807464. 
[24] C. S. Kim, Y. G. Kim, C.-D. Lü, and T. Morozumi, Azimuthal angle distribution in $B \rightarrow K^{*}(\rightarrow K \pi) \ell^{+} \ell^{-}$at low invariant $m\left(\ell^{+} \ell^{-}\right)$region, Phys. Rev. D $62(2000) 034013$ hep-ph/0001151; C. S. Kim, Y. G. Kim, and C.-D. Lü, Possible supersymmetric effects on angular distributions in $B \rightarrow K^{*}(\rightarrow K \pi) \ell^{+} \ell^{-}$decays, Phys. Rev. D $64(2001) 094014$ hep-ph/0102168.

[25] A. Faessler, T. Gutsche, M. A. Ivanov, J. G. Körner, and V. E. Lyubovitskij, The exclusive rare decays $B \rightarrow K\left(K^{*}\right) \bar{\ell} \ell$ and $B_{c} \rightarrow D\left(D^{*}\right) \bar{\ell} \ell$ in a relativistic quark model, Eur. Phys. J. Direct. C 4 (2002) 18 hep-ph/0205287.

[26] A. Ali and A. S. Safir, Helicity analysis of the decays $B \rightarrow K^{*} \ell^{+} \ell^{-}$and $B \rightarrow \rho \ell \nu_{\ell}$ in the large energy effective theory. Eur. Phys. J. C 25 (2002) 583 hep-ph/0205254.

[27] J. Charles, A. Le Yaouanc, L. Oliver, O. Pène, and J.-C. Raynal, Heavy to light form-factors in the heavy mass to large energy limit of QCD, Phys. Rev. D 60 (1999) 014001 hep-ph/9812358]; Heavy to light form-factors in the final hadron large energy limit: Covariant quark model approach, Phys. Lett. B 451 (1999) 187 hep-ph/9901378. See also M. J. Dugan and B. Grinstein, Phys. Lett. B 255 (1991) 583.

[28] M. Beneke and T. Feldmann, Symmetry-breaking corrections to heavy-to-light B meson form factors at large recoil, Nucl. Phys. B 592 (2001) 3 hep-ph/0008255.

[29] B. Stech, Form-factor relations for heavy to light transitions, Phys. Lett. B 354 (1995) 447 hep-ph/9502378; ; J. M. Soares, Form-factor relations for heavy to heavy and heavy to light meson transitions, Phys. Rev. D 54 (1996) 6837 hep-ph/9607284; Form factor relations for pseudoscalar to vector meson transitions, hep-ph/9810402.

[30] J. M. Soares, Form factor relations for heavy-to-light meson transitions: tests of the Quark Model predictions, hep-ph/9810421.

[31] G. Burdman and G. Hiller, Semileptonic form-factors from $B \rightarrow K^{*} \gamma$ decays in the large energy limit, Phys. Rev. D 63 (2001) 113008 hep-ph/0011266.

[32] U. Egede, T. Hurth, J. Matias, M. Ramon and W. Reece, in preparation.

[33] A. Ali, T. Mannel and T. Morozumi, Forward backward asymmetry of dilepton angular distribution in the decay $b \rightarrow s \ell^{+} \ell^{-}$, Phys. Lett. B 273 (1991) 505.

[34] T. Feldmann and J. Matias, Forward-backward and isospin asymmetry for $B \rightarrow K^{*} \ell^{+} \ell^{-}$ decay in the Standard Model and in supersymmetry, J. High Energy Phys. 01 (2003) 74 hep-ph/0212158.

[35] M. Beneke, T. Feldmann and D. Seidel, Exclusive radiative and electroweak $b \rightarrow d$ and $b \rightarrow s$ penguin decays at NLO, Eur. Phys. J. C 41 (2005) 173 hep-ph/0412400.

[36] J. Foster, K. i. Okumura and L. Roszkowski, Probing the flavour structure of supersymmetry breaking with rare B-processes: A beyond leading order analysis, JHEP 0508 (2005) 094 hep-ph/0506146.

[37] J. A. Casas and S. Dimopoulos, Stability bounds on flavor-violating trilinear soft terms in the MSSM, Phys. Lett. B 387 (1996) 107 hep-ph/9606237. 
